OPEN ACCESS

Edited by:

Robert C. Froemke, New York University,

United States

Reviewed by:

Paul George Anastasiades, University of Bristol, United Kingdom Michael M. Kohl,

University of Glasgow, United Kingdom

*Correspondence: Stephen D. Van Hooser vanhooser@brandeis.edu

TORCID:

Alexandra S. Cao orcid.org/0000-0003-1758-7445

Stephen D. Van Hooser orcid.org/0000-0002-1112-5832

Received: 27 October 2021 Accepted: 29 December 2021 Published: 03 February 2022

Citation:

Cao AS and Van Hooser SD (2022) Paired Feed-Forward Excitation With Delayed Inhibition Allows High Frequency Computations Across Brain Regions. Front. Neural Circuits 15:803065. doi: 10.3389/fncir.2021.803065

\section{Paired Feed-Forward Excitation With Delayed Inhibition Allows High Frequency Computations Across Brain Regions}

\author{
Alexandra S. Cao ${ }^{1,2+}$ and Stephen D. Van Hooser ${ }^{1,2,3 * \dagger}$ \\ ${ }^{1}$ Department of Biology, Brandeis University, Waltham, MA, United States, ${ }^{2}$ Volen Center for Complex Systems, Brandeis \\ University, Waltham, MA, United States, ${ }^{3}$ Sloan-Swartz Center for Theoretical Neurobiology, Brandeis University, Waltham, \\ MA, United States
}

The transmission of high frequency temporal information across brain regions is critical to perception, but the mechanisms underlying such transmission remain unclear. Long-range projection patterns across brain areas are often comprised of paired feed-forward excitation followed closely by delayed inhibition, including the thalamic triad synapse, thalamic projections to cortex, and projections within the hippocampus. Previous studies have shown that these joint projections produce a shortened period of depolarization, sharpening the timing window over which the postsynaptic neuron can fire. Here we show that these projections can facilitate the transmission of high frequency computations even at frequencies that are highly filtered by neuronal membranes. This temporal facilitation occurred over a range of synaptic parameter values, including variations in synaptic strength, synaptic time constants, short-term synaptic depression, and the delay between excitation and inhibition. Further, these projections can coordinate computations across multiple network levels, even amid ongoing local activity. We suggest that paired feed-forward excitation and inhibition provide a hybrid signal-carrying both a value and a clock-like trigger - to allow circuits to be responsive to input whenever it arrives.

Keywords: paired feed-forward excitation/inhibition feed-forward, timing, cross-network computing, striate cortex, area 17, LGN

\section{INTRODUCTION}

In digital electronics, computations are synchronized by the presence of a digital clock trigger signal that indicates when each digital component should examine its inputs and perform a computation. The digital clock offers several advantages for digital circuits. First, it allows digital circuits to have short integration times because the components do not need to consider information that has arrived at its inputs long in the past. Second, this short integration time allows the digital circuit to operate at high temporal speeds (many computations per second). Third, the digital clock allows synchronization of computations across different layers of digital components, because the arrival of related inputs is coordinated by the digital clock. 
In neuronal circuits, several properties would seem to make coordinated and precise high-speed computation across layers impossible: the apparent lack of an equivalent clock, the electrical filtering that is performed by the cell's membrane, and relatively slow synaptic time constants (von Neumann, 1963). However, we know that neural circuits do operate very quickly, as humans can make decisions within $200 \mathrm{~ms}$ of the arrival of a visual stimulus (Thorpe et al., 1996; Sherwin et al., 2012). In the retina, ganglion cells can respond very well to temporal frequencies as high as $50 \mathrm{~Hz}$ (Frishman et al., 1987), and LGN neurons can reliably follow the high temporal frequencies of retinal ganglion cell inputs (Movshon et al., 2005), despite postsynaptic membrane filtering and synaptic depression. Further, in natural environments, stimuli may arrive at any time, at slow rates or fast rates, and circuitry must be ready to respond quickly regardless of when a previous stimulus arrived. Therefore, the brain must have mechanisms for quickly following high frequency inputs.

Many long-range connections across brain areas exhibit a peculiar motif: long-range feed-forward excitatory input is paired, after a short delay, with a feed-forward inhibitory copy of the same input. At the retinogeniculate synapse, this inhibitory input is produced at a specialized dendro-dendritic synapse (the triad synapse) so that each feed-forward excitatory input is followed by a very fast and local inhibitory copy (Cox et al., 1998; Chen and Regehr, 2000; Blitz and Regehr, 2003, 2005). In other connections, such as those from the thalamus to cortex, across regions of the hippocampus, or interareal connections, feed-forward excitatory inputs project to both principal neurons and inhibitory interneurons, and the delayed inhibitory input onto principal neurons arises from the interneurons that received feed-forward excitation (Buzsaki, 1984; Agmon and Connors, 1991; Swadlow and Gusev, 2000; Porter et al., 2001; Swadlow, 2003; Gabernet et al., 2005; Pouille et al., 2009; Yang et al., 2013; Rock and Apicella, 2015; Bhatia et al., 2019).

It has been previously recognized that the coordinated arrival of the excitatory synaptic drive followed by the inhibitory drive can result in very temporally precise postsynaptic action potentials and short integration windows (Pouille and Scanziani, 2001; Wehr and Zador, 2003; Blitz and Regehr, 2005; Mittmann et al., 2005; Higley and Contreras, 2006; Kremkow et al., 2010b; Cardin, 2018). Here in theoretical work, we explore the basic physics of paired feed-forward excitation and inhibition. We show that paired feed-forward excitation and inhibition (FFEI) allows computation at very high temporal frequencies that "break" the membrane time constant barrier. Further, we show that this motif can synchronize computations across multiple hierarchical regions even when these regions have their own noisy ongoing local activity. Finally, we compare the limits of the temporal computations that are permitted in a low fan-in situation (1 paired synaptic input, such as at the triad synapse) and a high fan-in situation (such as in input to the cortex).

High temporal frequency transmission was possible over a wide range of synaptic parameters, including variations in synaptic strengths, synaptic time constants, synaptic depression, and delays between excitation and inhibition, suggesting that high temporal frequency transmission should be a characteristic of a variety of circuits that have this input structure. We conclude that paired feed-forward excitatory and delayed inhibitory input is a hybrid signal, providing information about the value of the input while at the same time imposing a clock-like trigger signal that shortens temporal integration in a manner that allows high frequency computation and synchronization across networks or layers.

\section{MATERIALS AND METHODS}

Neural circuit simulations were performed in MATLAB (MathWorks, Natick, MA). Leaky integrate and fire neurons were modeled with the following differential equation, using the forward Euler method with a $0.1 \mathrm{~ms}$ time step (Lapicque, 1907; Abbott, 1999):

$$
\frac{\mathrm{d} V_{m}}{\mathrm{~d} t}=-\frac{\left(V_{m}-V_{e}\right)}{\tau_{m}}-\frac{R_{m}}{\tau_{m}} \sum_{n} \alpha_{n} P_{n}(t)\left(V_{m}-V_{s y n, n}\right)
$$

where $V_{m}$ is the membrane potential of the neuron, $V_{e}$ is the leak potential, $\tau_{m}$ is the membrane time constant, $R_{m}$ is the membrane resistance, $P_{n}(t)$ is the synaptic conductance from synaptic input $n, V_{\text {syn, } n}$ is the resting potential at the synapse for input $n$, and $\alpha_{n}$ (= 1 for excitatory synapses) is a scaling coefficient for the synaptic current from input $n$. When $V_{m}$ reaches the threshold potential $V_{\text {thresh }}$, the model neuron generates a spike and $V_{m}$ is set to the reset potential $V_{\text {reset }}$ (see Tables $\mathbf{1}$ and $\mathbf{2}$ for values).

Synapses were modeled as a difference of exponentials, with two time constants (Dayan and Abbott, 2001):

$$
\begin{aligned}
& P_{n}(t)=\sum_{j} P_{\text {max }, n} B_{n}\left(\exp \left(-\frac{\Delta t_{j}}{\tau_{\text {fall }, n}}\right)-\exp \left(-\frac{\Delta t_{j}}{\tau_{\text {rise }, n}}\right)\right) \\
& B_{n}=\left(\left(\frac{\tau_{\text {rise }, n}}{\tau_{\text {fall }, n}}\right)^{\left(\frac{\tau_{\text {rise }, n}}{\tau_{\text {fall }, n}-\tau_{\text {rise }, n}}\right)}-\left(\frac{\tau_{\text {rise }, n}}{\tau_{\text {fall }, n}}\right)^{\left(\frac{\tau_{\text {fall }, n}}{\tau_{\text {fall }, n}-\tau_{\text {rise }, n}}\right)}\right)^{-1}
\end{aligned}
$$

where $P_{\max , n}$ is the maximum conductance of the synapse, $B_{n}$ is a normalization factor to ensure that the peak value of $P_{n}(t)$ is $P_{\max , n}, \Delta t_{j}$ is the time between $t$ and the $j$ th spike of synaptic input $n, \tau_{\text {fall }, n}$ is the fall time constant, and $\tau_{\text {rise }, n}$ is the rise time constant (see Tables 1 and 2 for values).

\section{Simulating Inputs to Neural Circuits}

Temporal information was generated using a Poisson process with a rectified sinusoidal firing rate:

$$
r(t)=\operatorname{rectify}(P R \sin (2 \pi F t))
$$

where $P R$ is the peak firing rate set to $100 \mathrm{~Hz}$ for all simulations, $F$ is the input modulation frequency, and $\operatorname{rectify}(x)=0$ if $x<0$, and $\operatorname{rectify}(x)=x$ for $x \geq 0$. For each set of simulations, temporal information was presented to our simulated neural circuits for a fixed length of time, independent of $F$.

In some of our simulations, we provided random background activity as an additional excitatory synaptic input. This activity was modeled as $N_{\text {inputs }}$ noisy Poisson inputs with a uniform firing rate FR (see Table 1 for values). FR was calculated such that, for 
TABLE 1 | Parameters for triad synapse simulations.

\begin{tabular}{|c|c|c|c|c|c|}
\hline \multicolumn{6}{|c|}{ Default model parameters: $d t=0.1 \mathrm{~ms}$} \\
\hline \multicolumn{2}{|c|}{ Leaky integrate and fire neuron } & \multicolumn{2}{|c|}{ Synaptic transmission, paired feed-forward E/I } & \multicolumn{2}{|c|}{ Synaptic transmission, feed-forward E } \\
\hline Parameter & Value & Parameter & Value & Parameter & Value \\
\hline$R_{\mathrm{m}}$ & $10 \mathrm{M} \Omega^{\S}$ & $P_{\max , \mathrm{i}}$ & $1.21 \mu S^{\Uparrow}$ & $V_{\text {syn }}$ & $0 \mathrm{mV} \S$ \\
\hline$V_{\mathrm{e}}$ & $-75 \mathrm{mV} \S$ & $V_{\text {syn, } \mathrm{e}}$ & $0 \mathrm{mV} \S$ & $\tau_{\text {fall }}$ & $20 \mathrm{~ms} \|$ \\
\hline$V_{\text {reset }}$ & $-80 \mathrm{mV} \S$ & $V_{\text {syn }, i}$ & $-80 \mathrm{mV} \S$ & $\tau_{\text {rise }}$ & $1 \mathrm{~ms} \|$ \\
\hline \multirow{4}{*}{$V_{\text {thresh }}$} & $-40 \mathrm{mV} \S$ & $\tau_{\text {fall, }}$, & $20 \mathrm{~ms}^{\|}$ & & \\
\hline & & $\tau_{\text {rise, } \mathrm{i}}^{\mathrm{i}}$ & $1 \mathrm{~ms}^{\|}$ & & \\
\hline & & $\Delta$ & $1 \mathrm{~ms}^{\dagger}$ & & \\
\hline & & $\alpha$ & 1.25 & & \\
\hline
\end{tabular}

Figure 1 modifications:

Panels C-E: Paired feed-forward E/l:

\begin{tabular}{|c|c|c|}
\hline$\tau_{\text {fall, } \mathrm{i}}$ & $P_{\text {max, e }}$ & $P_{\max , \mathrm{i}}$ \\
\hline $25 \mathrm{~ms}$ & $0.883 \mu S^{\mathbb{I}}$ & $0.723 \mu S^{ף}$ \\
\hline $30 \mathrm{~ms}$ & $0.581 \mu S^{\natural}$ & $0.403 \mu S^{\text {श }}$ \\
\hline $50 \mathrm{~ms}$ & $0.222 \mu S^{\pi}$ & $0.096 \mu S^{\Uparrow}$ \\
\hline
\end{tabular}

Panel F:

Paired feed-forward E/I

Feed-forward E

$\boldsymbol{P}_{\text {max, e }}$

$0.498 \mu S^{\text {शी }}$

$0.911 \mu \mathrm{S}$ ?

$1.21 \mu S^{\prime \prime}$

$1.46 \mu S^{\prime}$

$1.59 \mu S^{\prime \prime}$

$$
\begin{aligned}
& \boldsymbol{P}_{\text {max }, \mathrm{i}} \\
& 0.498 \mu S^{\text {I }} \\
& 0.911 \mu S^{\text {I }} \\
& 1.21 \mu \mathrm{S}^{\text {ก }} \\
& 1.46 \mu \mathrm{S}^{\text {? }} \\
& 1.59 \mu \mathrm{S} \text { " }
\end{aligned}
$$

\section{$\boldsymbol{P}_{\text {max }}$}

$0.032 \mu S^{\text {"ी }}$

$0.054 \mu S^{\text {"l }}$

$0.080 \mu S^{\text {f }}$

$0.120 \mu \mathrm{S}$ $0.160 \mu S^{\text {? }}$

Figure 2 modifications:

\begin{tabular}{|c|c|c|}
\hline Parameter & Value & \\
\hline $\begin{array}{l}N_{\text {inputs }} \\
F R \\
P_{\max , \text { noise }} \\
\tau_{\text {fall, noise }} \\
\tau_{\text {rise, noise }}\end{array}$ & $\begin{array}{l}50 \\
31.83 \mathrm{~Hz}^{\dagger \dagger} \\
2.26 \mathrm{nS}^{\& \&} \\
20 \mathrm{~ms}^{\|} \\
1 \mathrm{~ms}^{\|}\end{array}$ & \\
\hline \multicolumn{3}{|c|}{ Pane/s B-E: Paired feed-forward E/I: $d t=0.02 \mathrm{~ms}$} \\
\hline 1 & $0.597 \mu S^{\Uparrow}$ & $0.597 \mu S^{\mathbb{1}}$ \\
\hline 1.25 & $1.67 \mu S^{\uparrow}$ & $1.67 \mu S^{\Uparrow}$ \\
\hline 1.5 & $3.19 \mu \mathrm{S}$ & $3.19 \mu \mathrm{S} \eta$ \\
\hline 1.75 & $4.78 \mu \mathrm{S}^{\Uparrow}$ & $4.78 \mu \mathrm{S}^{\Uparrow}$ \\
\hline 2 & $5.58 \mu \mathrm{S}^{\Uparrow}$ & $5.58 \mu S^{\Uparrow}$ \\
\hline 3 & $4.46 \mu \mathrm{S}^{\Uparrow}$ & $4.46 \mu S^{\Uparrow}$ \\
\hline 5 & $3.78 \mu S^{\pi}$ & $3.78 \mu \mathrm{S}^{\Uparrow}$ \\
\hline
\end{tabular}

Panels B-D: Current injection amplitude $=8.38 \mathrm{nA}^{\text {q }}$

Panels E-G: Feed-forward E:

\begin{tabular}{lll}
\hline $\boldsymbol{\tau}_{\mathbf{m}}$ & $\boldsymbol{R}_{\mathbf{m}}$ & $\boldsymbol{P}_{\max }$ \\
\hline $7.5 \mathrm{~ms}$ & $7.5 \mathrm{M} \Omega$ & $0.090 \mu \mathrm{S}^{\text {" }}$ \\
$5 \mathrm{~ms}$ & $5 \mathrm{M} \Omega$ & $0.110 \mu \mathrm{S}^{\|}$ \\
$2 \mathrm{~ms}$ & $2 \mathrm{M} \Omega$ & $0.195 \mu \mathrm{S}^{\|}$ \\
$1 \mathrm{~ms}$ & $1 \mathrm{M} \Omega$ & $0.314 \mu \mathrm{S}^{\pi}$ \\
\hline
\end{tabular}

Figure 3 modifications:

Default noisy input parameters 
TABLE 1 | Continued

Panel F: Paired feed-forward E/l (noise): $d t=0.02 \mathrm{~ms}$

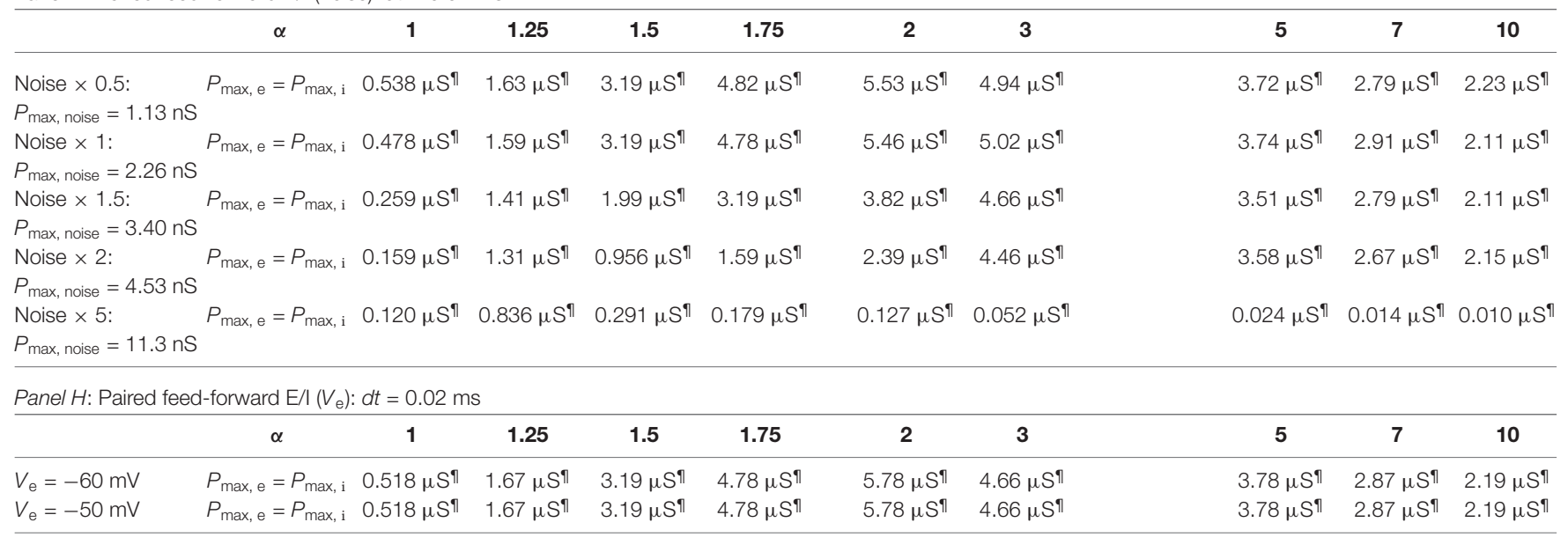

\section{Figure $\mathbf{4}$ modifications:}

Panels $B-D$ : Paired feed-forward E/l:

\begin{tabular}{|c|c|c|}
\hline$\Delta$ & $P_{\max , \mathrm{e}}$ & $\boldsymbol{P}_{\max , \mathrm{i}}$ \\
\hline $5 \mathrm{~ms}$ & $0.328 \mu S^{ף}$ & $0.328 \mu S^{q}$ \\
\hline $10 \mathrm{~ms}$ & $0.204 \mu S^{ף}$ & $0.204 \mu S^{q}$ \\
\hline
\end{tabular}

Figure 5 modifications (noise):

Panel A:

Paired feed-forward E/I

Feed-forward E

Noise only

Panels B-D, J-K:

Paired feed-forward $E / /$

Feed-forward $\mathrm{E}$

Noise only

Panel E:

Paired feed-forward E/I

$P_{\max , \mathrm{e}}=P_{\max , \mathrm{i}}=0.359 \mu \mathrm{S}$ "

$P_{\max }=0.016 \mu \mathrm{S}^{\pi}$

$P_{\max }=0$

$P_{\max , \mathrm{e}}=P_{\max , \mathrm{i}}=0.717 \mu \mathrm{S}$ ?

$P_{\max }=0.032 \mu S^{\text {I }}$

$P_{\text {max }}=0$

Feed-forward $\mathrm{E}$

\begin{tabular}{|c|c|c|}
\hline$P_{\max , \mathrm{e}}$ & $\boldsymbol{P}_{\max , \mathrm{i}}$ & $\boldsymbol{P}_{\max }$ \\
\hline $0.359 \mu S^{\Uparrow}$ & $0.359 \mu S^{\Uparrow}$ & $0.016 \mu S^{ף 1}$ \\
\hline $0.595 \mu S^{q}$ & $0.595 \mu S^{ף 1}$ & $0.024 \mu S^{ף}$ \\
\hline $0.717 \mu S^{\uparrow}$ & $0.717 \mu S^{ף 1}$ & $0.032 \mu S^{ף}$ \\
\hline $0.799 \mu S^{\uparrow}$ & $0.799 \mu S^{\Uparrow}$ & $0.047 \mu S^{\Uparrow}$ \\
\hline $0.835 \mu \mathrm{S}$ & $0.835 \mu \mathrm{S}$ & $0.055 \mu \mathrm{S}$ \\
\hline \multicolumn{3}{|c|}{ Figure 6 modifications: $d t=0.02 \mathrm{~ms}$} \\
\hline \multicolumn{2}{|c|}{ Paired feed-forward mixed-sign } & $P_{\max , \mathrm{e}}=P_{\max , \mathrm{i}}=1.31 \mu \mathrm{S}^{\Uparrow}$ \\
\hline \multicolumn{2}{|c|}{ Feed-forward single-sign } & $P_{\max }=0.066 \mu \mathrm{S}^{\Uparrow}$ \\
\hline \multicolumn{3}{|c|}{ Figure 6 supplement modifications: $d t=0.02 \mathrm{~ms}$} \\
\hline \multicolumn{2}{|c|}{ Paired feed-forward mixed-sign } & $P_{\max , \mathrm{e}}=P_{\max , \mathrm{i}}=1.20 \mu \mathrm{S}^{\Uparrow}$ \\
\hline \multicolumn{2}{|c|}{ Feed-forward single-sign } & $P_{\max }=0.068 \mu \mathrm{S}^{\pi}$ \\
\hline
\end{tabular}

Default AMPA+NMDA/GABA-like parameters

\begin{tabular}{|c|c|c|c|}
\hline Parameter & AMPA & AMPA+NMDA & AMPA+NMDA/GABA-like \\
\hline$P_{\max , \text { AMPA }}$ & $0.679 \mu S^{9}$ & $0.051 \mu S^{\Uparrow}$ & $0.798 \mu S^{\Re}$ \\
\hline$\tau_{\text {fall, AMPA }}$ & $0.72 \mathrm{~ms}^{\dagger}$ & $0.72 \mathrm{~ms}^{\dagger}$ & $0.72 \mathrm{~ms}^{\dagger}$ \\
\hline$\tau_{\text {rise, AMPA }}$ & $0.7 \mathrm{~ms}^{\dagger}$ & $0.7 \mathrm{~ms}^{\dagger}$ & $0.7 \mathrm{~ms}^{\dagger}$ \\
\hline$P_{\max , \mathrm{NMDA}}$ & & $0.026 \mu S^{ף}$ & $0.399 \mu S^{\Uparrow}$ \\
\hline$\tau_{\text {fall, NMDA }}$ & & $100 \mathrm{~ms}^{\ddagger}$ & $100 \mathrm{~ms}^{\ddagger}$ \\
\hline$\tau_{\text {rise, NMDA }}$ & & $3.2 \mathrm{~ms}^{\ddagger}$ & $3.2 \mathrm{~ms}^{\ddagger}$ \\
\hline$\Delta$ & & & $1 \mathrm{~ms}^{\dagger}$ \\
\hline
\end{tabular}


TABLE 1 | Continued

Figure 8 modifications:

Default AMPA+NMDA/GABA $A+G A B A_{B}$ parameters

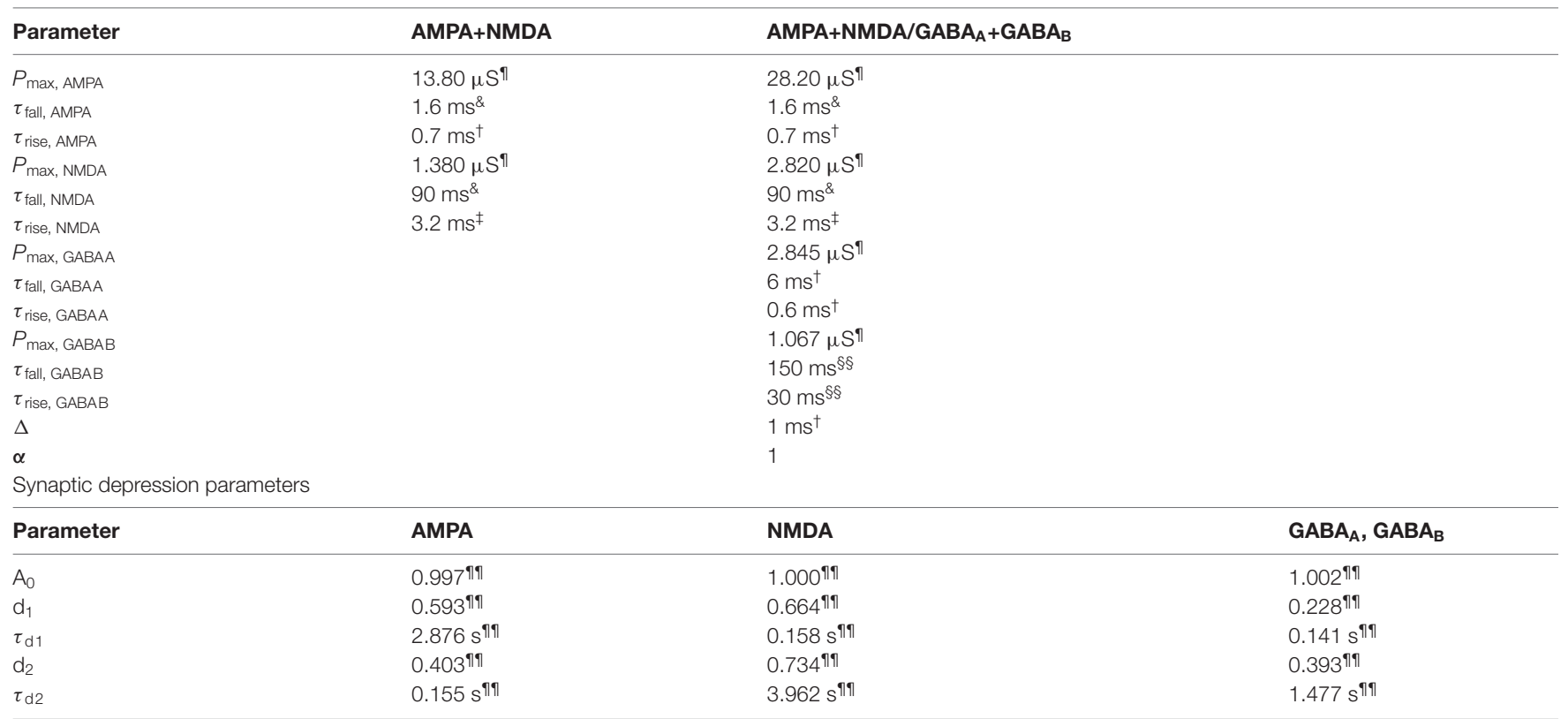

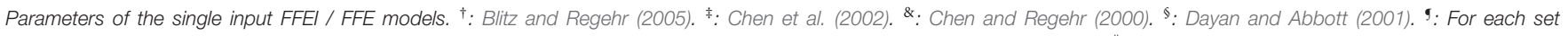
of simulations, synaptic input strengths were chosen to balance the response at $\mathrm{F}=5 \mathrm{~Hz}$ between different parameter sets." : Excitation at biological triad synapses involves a combination of a fast-decaying AMPA current (Chen et al., 2002; Blitz and Regehr, 2005) and a slow-decaying NMDA current (Chen et al., 2002), resulting in a complex excitatory conductance (Chen et al., 2002). In order to simplify the analysis of this circuit motif, synaptic time constants were chosen to produce a single excitatory current with medium decay. ${ }^{\dagger \dagger}$ : The background noise firing rate was adjusted such that the mean firing rates of the feed-forward inputs and each noisy input were balanced. \&\&: The strength of noisy input was adjusted such that the total noise was comparable to the feed-forward input. ${ }^{\circledR}:$ GABA $A_{A}$ and $G A B A_{B}$ channels were both included in triad synapse models with depression (Soltesz and Crunelli, 1992); $G A B A_{B}$ time constants were chosen in the range of experimental values (Thompson, 1994; Destexhe and Sejnowski, 1995). 99 : Depression parameters were obtained by fitting paired-pulse ratio data from the retinogeniculate synapse (Chen et al., 2002; Blitz and Regehr, 2005) to a depression model with two depressing factors (Varela et al., 1997).

each noisy input, the probability of seeing 1 background spike during an input cycle is equal to the probability of a feed-forward input spike over one cycle (that is, the mean firing rate of the feed-forward inputs and each noisy input were balanced):

$$
F R=\frac{P R}{\pi}
$$

\section{Calculating the Power of Spiking Responses at a Particular Frequency}

Spiking responses were converted to a firing rate per time step $(\mathrm{Hz})$, and the following equation was used to calculate the Fourier coefficient magnitude at a particular frequency (Crawford, 1968):

$$
F C_{F}=\left|\frac{2 \Delta t}{L} * \sum_{t_{\text {index }}=0}^{L / \Delta t} R(t) \exp (-2 \pi i * F * t)\right|, t=t_{\text {index }} \Delta t
$$

where $\Delta t$ is the time step size in seconds, $L$ is the length of the spiking response in seconds, $t_{\text {index }}$ is time as an integer index, $R(t)$ is the spiking response in $\mathrm{Hz}$, and $F$ is a particular frequency.

To normalize the power of spiking responses at a particular frequency, we used the average power calculated over all frequencies $\left(F C_{\text {avg }}\right)$ as a normalization factor. For a time interval of length $L$ in time steps of $\Delta t, 1 / \Delta t$ and $1 / L$ are the maximum and minimum frequencies that can be represented, respectively. Therefore, $F C_{\text {avg }}$ was determined by averaging $F C_{F}$ over $F=[0,1 / \Delta t]$ in intervals of $1 / L$. The normalized frequency response was then expressed as $F C_{F} / F C_{\text {avg. }}$. In cases where $F C_{\text {avg }}=0$, the normalized frequency response was set to 0 .

Unless otherwise stated, $F C_{F}, F C_{\text {avg }}$, and $F C_{F} / F C_{\text {avg }}$ were each averaged over 10 trials for each model.

\section{Balancing Excitation and Inhibition in Triad Synapse Models}

When varying the inhibitory fall time constant $\left(\tau_{f a l l, i}\right)$ in our triad synapse models, we scaled $P_{\max , i}$ relative to $P_{\max , e}$, such that the total excitation and inhibition are balanced:

$$
P_{\max , i}=P_{\max , e} \frac{B_{e}}{B_{t}} \frac{\tau_{f a l l, e}-\tau_{\text {rise }, e}}{\tau_{f a l l, i}-\tau_{\text {rise }, i}}
$$

The above equation was derived by first integrating $P_{e}(t)$ and $P_{i}(t)$ for a single spike at $t=0$ over the interval $t=[0, \infty]$, as a measure of the total conductance due to a single spike. $P_{\max , i}$ values were then obtained by setting the total excitation equal to the total inhibition, and then solving for $P_{\max , i}$ given a set of time constants and excitatory parameters. 
TABLE 2 | Parameters for LGN-cortex simulations.

\begin{tabular}{llc}
\hline & & Default model parameters: $\boldsymbol{d} \mathbf{t}=\mathbf{0 . 1} \mathbf{~ m s}$ \\
\hline Parameter & Leaky integrate and fire neuron \\
\hline$\tau_{\mathrm{m}}$ & Interneuron (I) & Cortical neuron (E) \\
$R_{\mathrm{m}}$ & $10 \mathrm{~ms}^{\S}$ & $10 \mathrm{~ms}^{\S}$ \\
$V_{\mathrm{e}}$ & $10 \mathrm{M} \Omega^{\S}$ & $10 \Omega^{\S}$ \\
$V_{\text {reset }}$ & $-75 \mathrm{mV}$ & $-75 \mathrm{mV}^{\S}$ \\
$V_{\text {thresh }}$ & $-80 \mathrm{mV} \S$ & $-80 \mathrm{mV}^{\S}$ \\
\hline
\end{tabular}

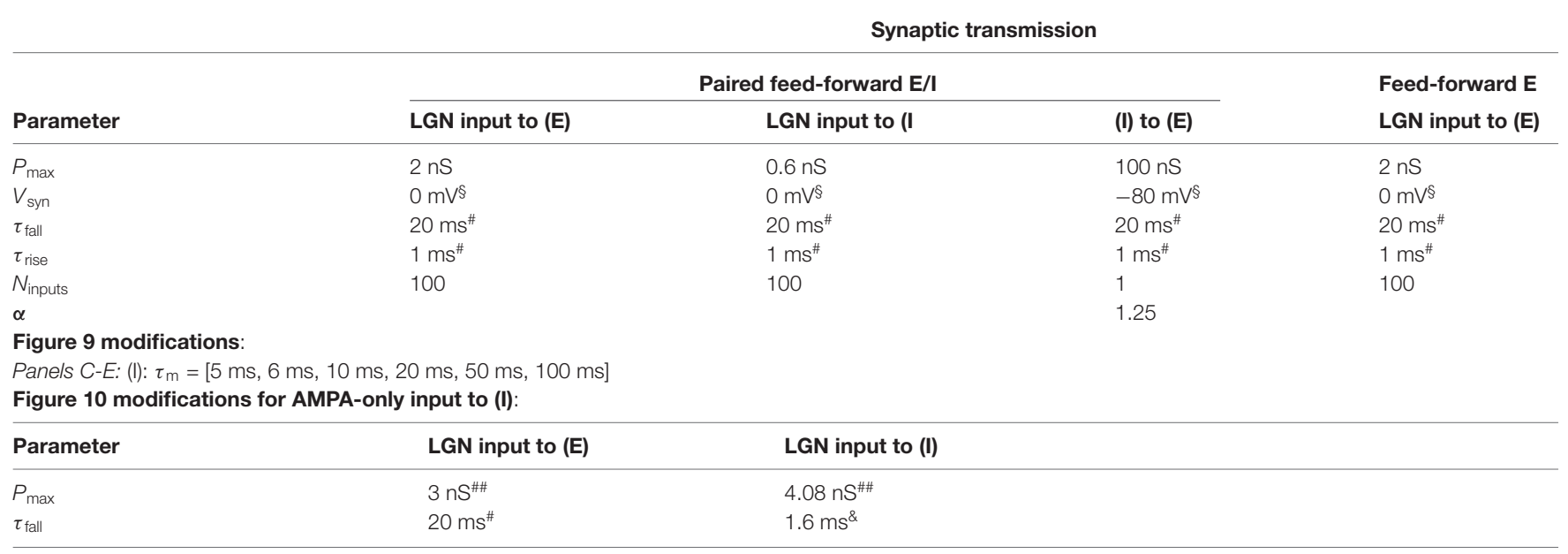

Parameters of the many input FFEI / FFE models. \#: Stratford et al. (1996). $\diamond$ : The firing threshold of inhibitory interneurons was lowered, in order to represent the increased excitability of these neurons compared to other neurons in the cortex (McCormick et al., 1985). "\#: Synaptic input strengths were chosen to balance the (I) and (E) response at $F=5 \mathrm{~Hz}$ between the new parameter set and the default parameters. See additional annotations for constants chosen in Table 1 caption.

\section{Determining the Expected Output of an Exclusive-or Computation}

In Figure 6, we examine the output of a circuit designed to perform an exclusive-or (XOR) computation of two inputs. The expected output of the circuit was determined by performing the XOR computation on sliding windows of length $t_{b i n}=10 \mathrm{~ms}$ or $5 \mathrm{~ms}$. If only one of the inputs fires during the time interval $\left[t-\frac{t_{\text {bin }}}{2}, t+\frac{t_{\text {bin }}}{2}\right]$, then output is expected at time $t$.

\section{Simulating Depression at the Retinogeniculate Synapse}

Paired-pulse ratio data for AMPA and NMDA (Chen et al., 2002) and for GABA (Blitz and Regehr, 2005) in the retinogeniculate synapse were fitted to the following depression model (Varela et al., 1997):

$$
A=A_{0} D_{1} D_{2}
$$

In the above equation, $A$ is a scaling factor on the amplitude of the synaptic current generated by each presynaptic spike, $A_{0}$ is the undepressed scaling factor, and both $D_{1}$ and $D_{2}$ are depressing factors with default values of 1 . Following each presynaptic spike, $D_{i}$ is set to $D_{i} d_{i}$, and $D_{i}$ decays back to 1 with a time constant $\tau_{d_{i}}$ (see Table 1 for values and Supplementary Figure 3 for fits).

The code for the article is at https:/github.com/VHLab/vhlab-ffei-matlab.

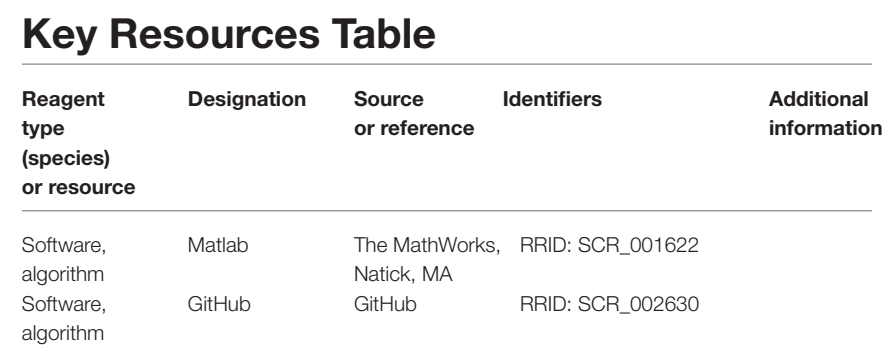

\section{Sex as a Variable}

Not applicable to the computational models studied here.

\section{RESULTS}

Our goal was to understand the physics of the configuration of paired feed-forward excitatory and inhibitory (FFEI) connections that are commonly observed in projections across brain regions. We analyzed the problem in two regimes. First, we analyzed a low convergence situation $\left(\mathrm{N}_{\text {inputs }}=\sim 1\right)$, inspired by the triad retinogeniculate synapse in the LGN. Here, we found that FFEI projections allowed feed-forward computations at much higher frequencies than if feed-forward excitatory (FFE) projections were employed alone. In order to focus specifically on the difference between FFEI projections and FFE projections, we first explored models that were inspired by 
A

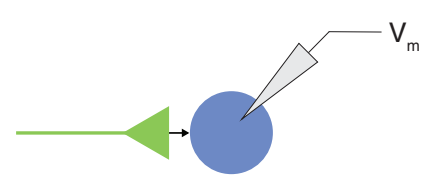

Input rate

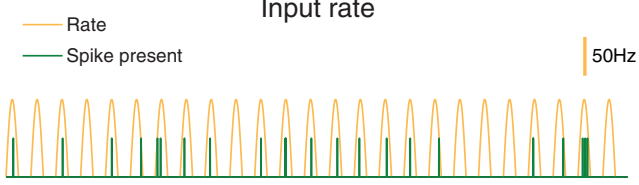

Postsynaptic conductance

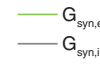
$G_{\text {syn, }, i}$

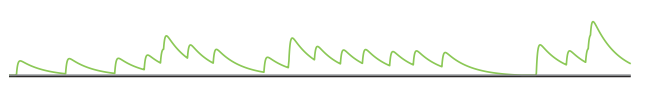

Postsynaptic response

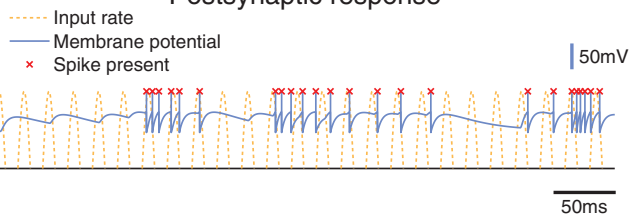

B

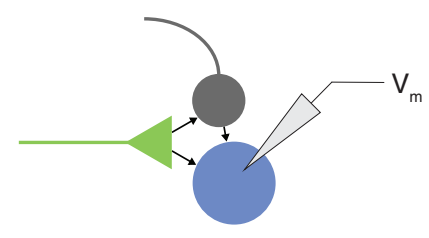

Input rate

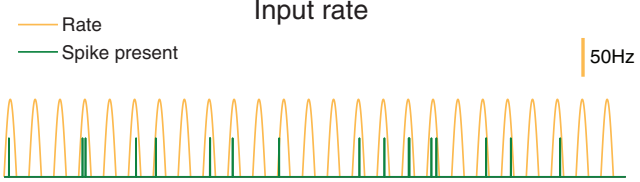

Postsynaptic conductance

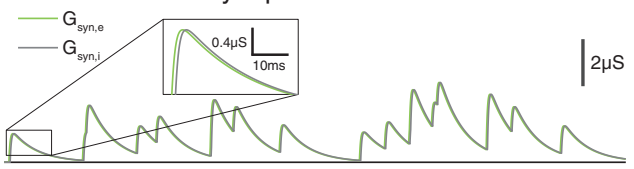

Postsynaptic response

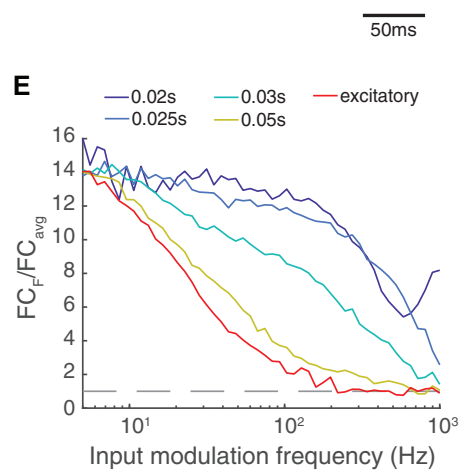

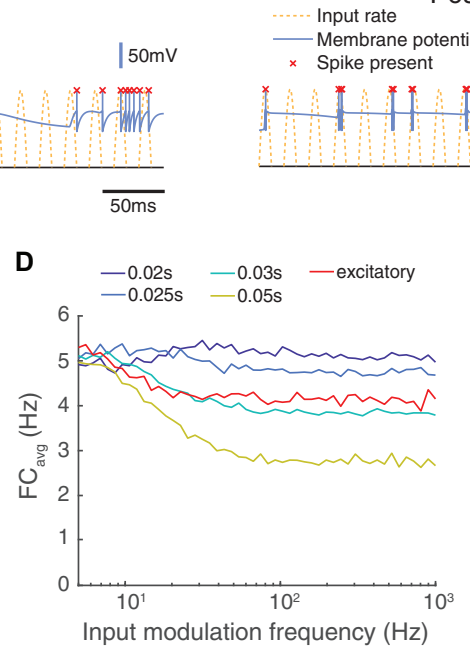

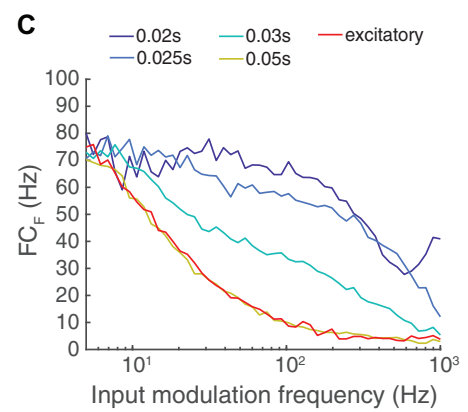

$\mathbf{F}$

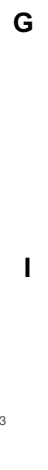

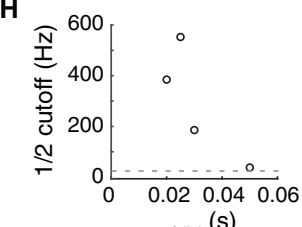

fall, $(\mathrm{s})$

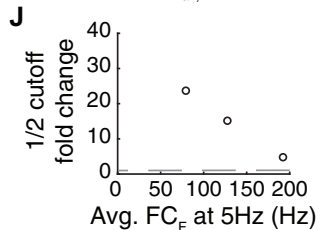

FIGURE 1 | Paired feed-forward excitation and delayed inhibition in a triad synapse model allows transmission of higher frequencies than feed-forward excitation alone. (A) Top: A feed-forward excitatory (E) model circuit with a single RG-like cell (light green) providing excitatory input to an LGN-like neuron (blue). Bottom: A $0.5 \mathrm{~s}$ simulation of the model circuit with a rectified sinusoidal input firing rate (yellow) with a peak rate (PR) of $100 \mathrm{~Hz}$ and an input modulation frequency (F) of $50 \mathrm{~Hz}$; presynaptic spikes were generated with a Poisson process (dark green). The postsynaptic conductance (light green) and the membrane potential of the LGN cell (blue) are simulated in response to the feed-forward input. Output firing times are marked (red x), and the postsynaptic response is compared to the underlying input characteristics (dashed yellow). (B) Same, but for a paired feed-forward excitatory/inhibitory (E/l) model circuit with a single feed-forward cell (light green) providing excitatory input to an output neuron (blue) and to an interneuron (gray), which in turn provides delayed inhibitory input (1 ms) to the output neuron. (C) For a range of input modulation frequencies $F$, the Fourier coefficient of the output at $F\left(F C_{F}\right)$ was averaged over 10 trials for triad synapse models with different inhibitory

(Continued) 
FIGURE 1 | Continued

conductance characteristics. Legend entries indicate the length of the inhibitory time constant $\tau_{\text {fall, }}$ for each paired feed-forward $E / I$ model; "excitatory" indicates the feed-forward E model. Synaptic strengths were adjusted so that Fourier coefficients at input modulation $5 \mathrm{~Hz}$ were approximately $75 \mathrm{~Hz}$. (D) Mean Fourier coefficient of the output over all frequencies, including those that are much higher and lower than the modulation frequency. (E) Fourier coefficient of the output at $\mathrm{F}$ divided by the mean overall power $\left(F C_{F} / F C_{\text {avg }}\right)$. Dashed line indicates $F C_{F} / F C_{\text {avg }}=1$. $(\mathbf{F})$ Same as $\mathrm{C}$, except that synaptic strength $\mathrm{P}_{\max }$ was adjusted to produce a range of Fourier coefficient values at an input modulation frequency at $5 \mathrm{~Hz}$ (weak to strong: red to dark blue). Solid lines indicate the feed-forward E/I model, and dashed lines indicate the feed-forward E model. In all cases, paired $\mathrm{E} / \mathrm{I}$ input resulted in greater transmission at high input modulation frequencies than $E$ input alone. (G) The influence of the inhibitory synaptic fall-off time constant $\tau_{\text {fall, }}$ on transmission $\mathrm{FC}_{F} / \mathrm{FC}_{\text {avg }}$, measured at $F=50 \mathrm{~Hz}$ (triangles) and $100 \mathrm{~Hz}$ (squares). Dashed lines indicate feed-forward $\mathrm{E}$ model at $50 \mathrm{~Hz}$ (top) and $100 \mathrm{~Hz}$ (bottom). The paired E/I configuration exhibits greater transmission over a range of inhibitory synaptic time constants. (H) The influence of the inhibitory synaptic fall-off time constant $\tau_{\text {fall, }}$ on the input modulation frequency at which $\mathrm{FC}_{\mathrm{F}} / \mathrm{FC}_{\text {avg }}$ is $\frac{1}{2}\left(\mathrm{FC}_{\mathrm{F}} / \mathrm{FC}_{\text {avg }}\right.$ at $\left.F=5 \mathrm{~Hz}\right)$. Dashed line indicates the $\frac{1}{2}$ cutoff for the feed-forward $E$ model. (I) For each paired feed-forward $E / I$ model shown in $(\mathbf{F})$, the $F C_{F}$ fold change with respect to the feed-forward $\mathrm{E}$ model of matching response at $F=5 \mathrm{~Hz}$ for $F=50 \mathrm{~Hz}$ (triangles) and $F=100 \mathrm{~Hz}$ (squares). Dashed line indicates a fold change of 1 . (J) For each paired feed-forward E/I model shown in $(\mathbf{F})$, the $\frac{1}{2}$ cutoff fold change with respect to the feed-forward $\mathrm{E}$ model of matching output power (denoted by line style and $\mathrm{FC}_{\mathrm{F}}$ at $F=5 \mathrm{~Hz}$ ) was calculated as FFEI/FFE. For the lowest firing rates, $\frac{1}{2}$ cutoff was greater than $1,000 \mathrm{~Hz}$ in the FFEl case (out of range). Dashed line indicates a fold change of 1 . In all, paired E/I input allowed transmission of higher input modulation frequencies over a wide range of inhibitory synaptic time constants and for a wide range of synaptic input strengths.

the LGN triad synapse but were much simpler, to demonstrate the phenomena and how they depend on the inhibitory delay and properties of the inhibitory synapse. Next, we explored a more realistic model of the triad synapse that includes synaptic depression of excitatory and inhibitory synapses and showed that the principles of the very simple models apply under these conditions. Finally, we examined a high convergence situation ( $\left.\mathrm{N}_{\text {inputs }}=\sim 50-150\right)$ such as is found at thalamocortical and intercortical projections.

\section{Paired Feed-Forward Excitatory and Inhibitory Inputs Allow the Transmission of Temporal Information at High Frequencies}

We began with a simple model inspired by the retina-LGN triad synapse that is particularly common at synapses between retinal $\times$ cells and LGN $\times$ cells $($ Koch, 1985; Hamos et al., 1987; Lam et al., 2005; Bickford, 2019). At these synapses, excitatory retinal input is provided to an LGN neuron and to an adjacent terminal bouton of the dendrite of an interneuron. The interneuron dendrite, which is electrotonically isolated from its soma (Morgan and Lichtman, 2020), also contacts the LGN cell at very nearly the same location as the excitatory cell, providing an only slightly-delayed inhibitory input to the LGN neuron (Cox et al., 1998). To build our model, we included a feed-forward glutamatergic excitatory synaptic current with temporal dynamics that were set to be between the fast-decaying AMPA current and the slower-decaying NMDA current observed at triad synapses (Chen et al., 2002). Fast
GABAergic currents were included with a $1 \mathrm{~ms}$ delay, also following experimental measurements (Blitz and Regehr, 2003, 2005). While the total synaptic conductance was varied, the maximal conductances of the excitatory and inhibitory synapses were selected so that the total conductance (the area under the curve) of excitation and inhibition were identical; that is, the total integrated conductance of excitation and inhibition were balanced.

To assess the transmission of temporal information in this model, we simulated the spiking responses of a leaky integrateand-fire neuron to input spikes generated via a Poisson process with a rectified sinusoidal firing rate (Figures $\mathbf{1 A}, \mathbf{B}$ ). The rate at which spikes were generated varied in time sinusoidally from 0 spikes/s to 100 spikes/s and the frequency of the sinewave was termed the modulation frequency. At each $0.1 \mathrm{~ms}$, a random number (0-1) was drawn, and a spike was generated if the random number was less than the product of the rate and the bin size $(0.1 \mathrm{~ms})$. While the FFE configuration produces extra spikes that are outside the sinusoidal input modulation, output spikes in the FFEI input configuration are well locked to the sinusoidal input modulation. We examined the transmission of the FFEI projection over a range of inhibitory synapse fall time constants that varied from 20 to $50 \mathrm{~ms}$, and compared its transmission to the case where only feed-forward excitatory (FFE) input was provided. Because changing the fall time constant of the inhibitory synaptic current, while keeping integrated $\mathrm{E}$ and I conductance fixed, alters the fraction of the excitatory conductance that arrives earlier in the postsynaptic response, the overall responsiveness of the cell also varies as we adjusted the inhibitory synapse fall time constant. To compare equally responsive models, we adjusted the feed-forward synaptic weight so that each model generated a response of about $75 \mathrm{~Hz}$ when driven with an input modulating frequency of $5 \mathrm{~Hz}$.

FFEI projections demonstrated a remarkable ability to transmit high frequency information. To measure transmission quality at each input frequency, we calculated the Fourier coefficient (FC) of the spiking response at the input modulation frequency $\left(\mathrm{FC}_{\mathrm{F}}\right.$; Figure 1C). The Fourier coefficient is higher when spikes are well locked to the stimulus, and drops as the number of spikes fired per cycle becomes lower or becomes statistical (that is, with some missed cycles). Projections with only excitatory synapses were filtered strongly at input modulation frequencies ranging from 20 to $100 \mathrm{~Hz}$. In contrast, FFEI projections with inhibitory time constants of $20 \mathrm{~ms}$ transmitted with nearly equal fidelity from 5 to $100 \mathrm{~Hz}$, and did not reach a 50\% reduction in Fourier coefficient responses until the input modulation frequency reached about $400 \mathrm{~Hz}$.

While the Fourier coefficient at the input modulation frequency $\left(\mathrm{FC}_{\mathrm{F}}\right)$ measures the output transmission at that single frequency, it did not provide information about how much of the total output of the cell was concentrated exclusively at the input modulation frequency. For example, if the output cell increased its overall response only tonically, then $\mathrm{FC}_{\mathrm{F}}$ would still exhibit an increase. To examine the fraction of the output cell's total frequency response that was at the 
input modulation frequency, we divided $\mathrm{FC}_{\mathrm{F}}$ by the average Fourier coefficient calculated over all frequencies, including frequencies much higher and lower than the input modulation frequency (Figure 1D), to produce a normalized frequency response measure (Figure 1E). This analysis indicated that FFEI projections transmitted information that was highly specific to the input modulation frequency over a wide frequency range of $5-100 \mathrm{~Hz}$, while the specificity of information transmitted by excitatory-alone projections fell off rapidly with frequency.

The ability to provide specific output at the input modulation frequency was highly related to the inhibitory fall time constant. When the inhibitory fall time constant was short, then the advantage of the FFEI configuration was greater. When the time constant is small $(20 \mathrm{~ms})$, then the inhibition comes right on the heels of the excitation so that the excitation cannot drive the cell for a long time. When there is no inhibition, the excitation can drive the cell for a long time, which means less of the response is at the input modulation frequency. When there is a long time constant of inhibition $(50 \mathrm{~ms})$, then the inhibition is more spread out in time and the behavior is more intermediate, although more single feed-forward input spikes are missed by the output neuron than in the short time constant case (Supplementary Figure 1).

We plotted the normalized output responses for input modulation frequencies of 50 and $100 \mathrm{~Hz}$ in Figure 1G. For an inhibitory fall time constant of $20 \mathrm{~ms}$, the postsynaptic neuron produced output at the input modulation frequency that was more than 12 times the amount of average output at other frequencies. As the inhibitory fall time constant was increased (and weight compensated to retain equal responsiveness), the output neuron behaved more like the excitatory-only configuration, as one would expect. We quantified the ability of the synapse to maintain its response at high temporal frequencies by identifying the temporal frequency where the output was reduced to an arbitrary threshold of $\frac{1}{2}$ its value at $5 \mathrm{~Hz}$ (a physiologically meaningful threshold would depend on the noise of each circuit). The $\frac{1}{2}$ cutoff frequency was also highly dependent on the inhibitory fall time constant (Figure 1H).

It was possible that the relative advantage of FFEI transmission compared to FFE was not general, but instead was specific to the particular levels of synaptic input strengths that were chosen in order to drive output responses of about $75 \mathrm{~Hz}$ at a $5 \mathrm{~Hz}$ input modulation frequency. To examine this possibility, we compared FFEI and FFE models that were matched at $5 \mathrm{~Hz}$ input modulation but over a wide variety of output response levels (Figure 1F). In all cases, the FFEI projection responses at the input modulation frequency were much stronger than those of the FFE projection over a wide range of input modulation frequencies. To quantify these changes, we examined the fold change of transmission of FFEI compared to FFE at input modulation frequencies of 50 or $100 \mathrm{~Hz}$ for these different levels of synaptic drive (Figure 1I). For all of these levels of synaptic drive, the FFEI projection produced at least two times as much drive at 50 and $100 \mathrm{~Hz}$ compared to FFE alone. The $\frac{1}{2}$ cutoff frequency was also more than four times higher for FFEI compared to FFE (Figure 1J).

\section{Paired Feed-Forward Excitatory and Inhibitory Inputs Allow Effective Transmission at Frequencies That Are Highly Filtered by the Output Cell's Membrane Properties}

The ability of the output cell to reliably follow very high input frequencies was surprising because the membrane properties of neurons act as a low-pass filter, and high frequency current fluctuations are not well transmitted to membrane voltage. We compared the ability of the output neuron to follow synaptic input provided through either FFEI or FFE projections with the ability of the output neuron to follow direct sinusoidal membrane current injections of a fixed amplitude (Figure 2).

As before, we calibrated the output of the neuron to hit a target rate $(75 \mathrm{~Hz})$ at a $5 \mathrm{~Hz}$ input modulation frequency. For the FFEI and FFE simulations, we did this by setting the synaptic weight, and for the current injection simulations, we did this by setting the amplitude of the sinusoidal current injection. We compared the raw Fourier coefficient measured at the input modulation frequency (Figure 2B), the overall response across all frequencies (Figure $2 \mathrm{C}$ ), and the normalized frequency response to measure the specificity of the output response to the input modulation frequency (Figure 2D). As expected, the cell followed the sinusoidal current injection with substantial fidelity until a critical frequency was reached, after which the input fluctuations became too small to generate action potentials. This resulted in a rapid drop in the ability of the cell to follow the output frequencies. By contrast, FFEI inputs continued to drive responses at high frequencies, well beyond the point at which the sinusoidal current could drive spikes. While the critical frequency at which spiking stops for direct current injection does depend upon the amplitude of the current used, the take-home point here is that FFEI projections drive responses at higher input frequencies when responses to FFEI and direct current injections are matched at low frequencies.

The addition of an inhibitory conductance in the FFEI case will necessarily reduce the effective membrane time constant, and one may ask whether the increased transmission is primarily due to the reduced time constant. To explore this, we modified the membrane time constant of our model neuron over a 10-fold range and examined the influence of the passive membrane time constant on the temporal frequency of transmission (Figures 2E-G). We observed very small influences of modifying the membrane time constant, indicating that a passive adjustment of the membrane time constant cannot account for increased high frequency transmission observed here.

At first glance, it seems almost paradoxical that inputs delivered via FFEI projections can apparently "break the limit" of the membrane time constant. To gain intuition into the 


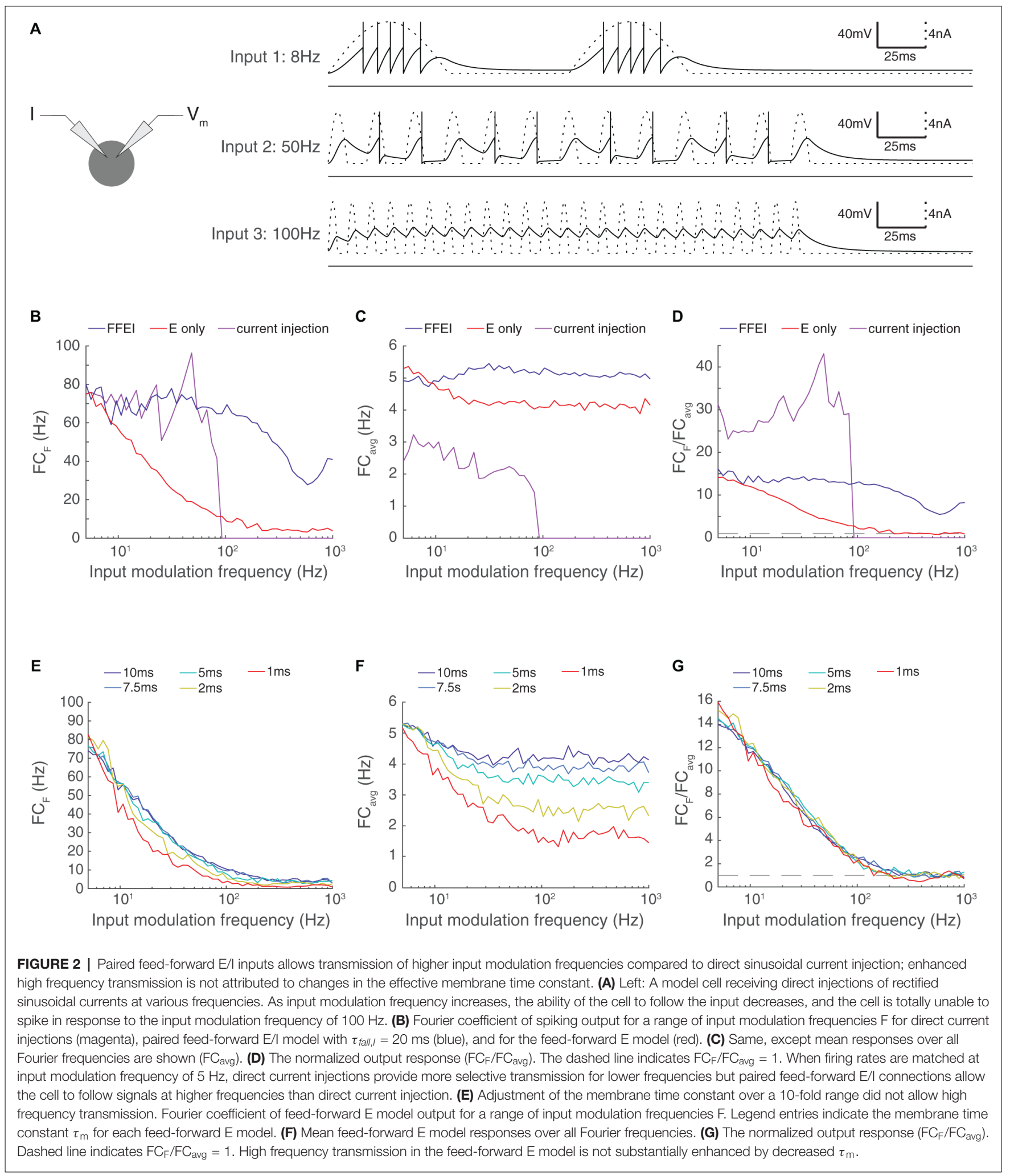

mechanism of action of FFEI projections, we examined synaptic currents generated by a $100 \mathrm{~Hz}$ train of input spikes when delivered either by FFE or FFEI projections (Figure 3A). To study the influence of different amounts of inhibition on transmission, we also varied the relative weight (total area under conductance curve per presynaptic spike) of the inhibitory conductance to the excitatory conductance and called this quantity $\alpha$. 


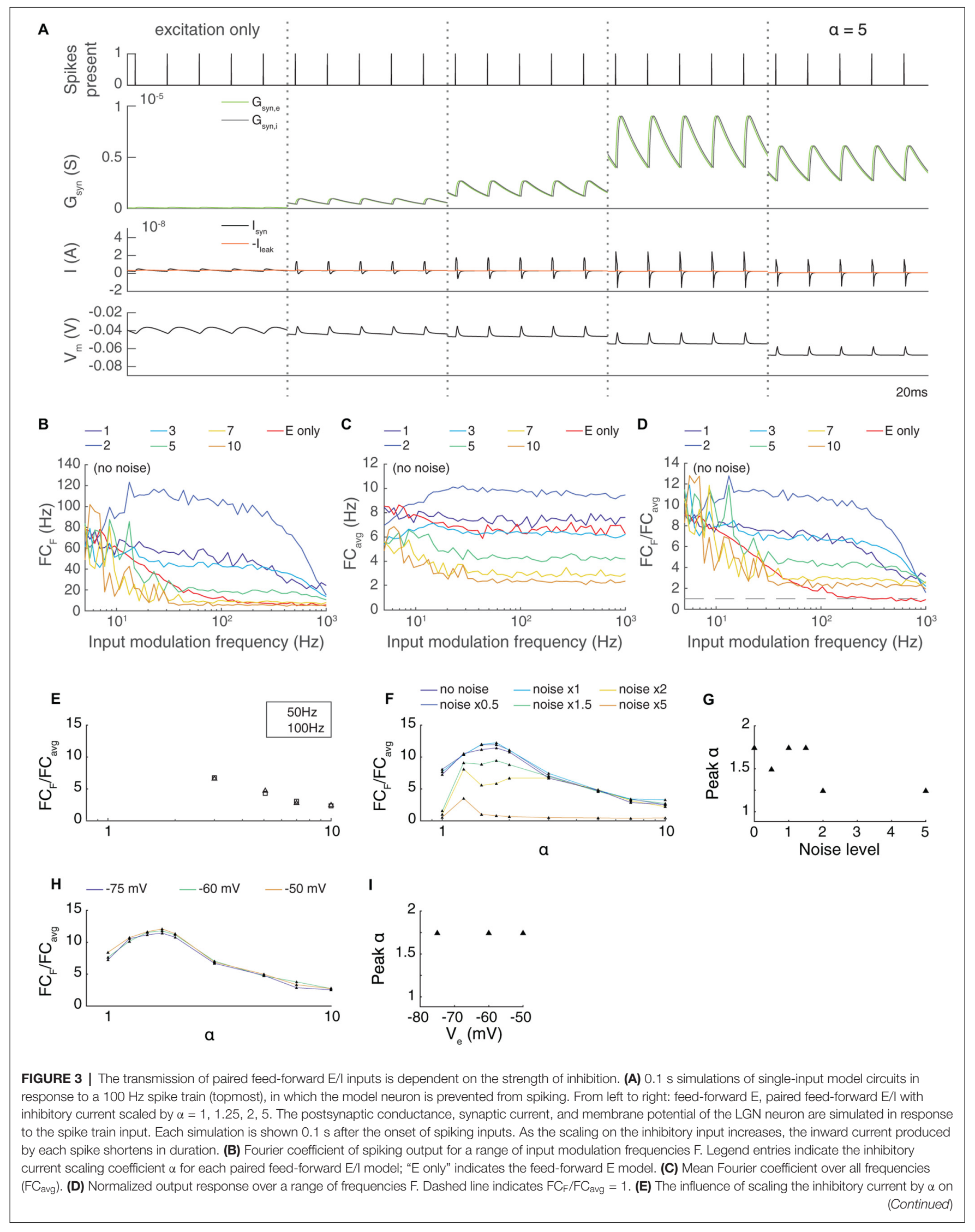




\section{FIGURE 3 | Continued}

transmission $\mathrm{FC}_{\mathrm{F}} / \mathrm{FC}_{\text {avg }}$, measured at $F=50 \mathrm{~Hz}$ (triangles) and $100 \mathrm{~Hz}$ (squares). Dashed lines indicate feed-forward $\mathrm{E}$ model at $50 \mathrm{~Hz}$ (top) and $100 \mathrm{~Hz}$ (bottom). The paired feed-forward E/I model exhibits greater transmission with stronger inhibition, plateauing at $\alpha=1.5$. (F) The influence of excitatory background noise on transmission $\mathrm{FC}_{\mathrm{F}} / \mathrm{FC}_{\text {avg }}$ with different levels of inhibitory scaling $\alpha$, measured at $F=50 \mathrm{~Hz}$. Legend entries indicate relative background current strength. (G) Inhibitory scaling coefficients $\alpha$ that produced the strongest transmission for each background noise level shown in $(F)$. For these models, moderate inhibition $(1.5 \leq \alpha \leq 2)$ produces greater transmission when excitatory background noise is weak. As background noise becomes stronger, the model performs best with slightly scaled inhibition ( $\alpha=1.25$ ). (H) The influence of scaling the inhibitory current at $50 \mathrm{~Hz}$ when the reversal potential of the leak current was increased, reducing the threshold. Unlike when noise was increased, there is no dependence of the transmission on the parameter $\alpha$. (I) Optimal values of $\alpha$ for different leak reversal potentials.

The synaptic currents and postsynaptic potentials generated by excitatory-only projections were highly blurred in time and exhibited substantial temporal summation compared to those delivered by FFEI projections. In order for output spike timing to be precise, the input must be very brief in time, and synapses with NMDA receptors cannot support this tight precision. FFEI projections delivered pulse-like current injections that were concentrated in time in a manner that varied with the total amount of inhibition $\alpha$. However, sharp, high frequency inputs are highly filtered by the neuronal membrane, so the brief input to the neuron must have very high amplitude. This can be produced by strong $\mathrm{E}$ and I synaptic weights, and the delay between the two serves to allow enough rapid current to produce spikes without causing a long-lasting trend in the membrane voltage. Therefore, while inputs at high frequencies are highly filtered, the large, sharp current pulses produced by the FFEI input configuration allow spiking transmission at those high frequencies despite the high filtering.

To examine the optimal amount of feed-forward inhibition, we plotted the transmission of feed-forward signals as a function of input modulation frequency (Figures 3B-D). As before, synaptic weights were set so that transmission at an input modulation of $5 \mathrm{~Hz}$ was equal to about $75 \mathrm{~Hz}$. Transmission at high frequencies was most efficacious when $\alpha$ was between 1.5 and 3. Larger values of $\alpha$ suppressed transmission because the level of inhibition was too high.

In many neural circuits, the ongoing activity provides a background input that might influence the feed-forward transmission of information. To investigate how these background signals might influence the level of inhibition needed for optimal feed-forward transmission, we modeled this background input as 50 noisy independent Poisson inputs, with varying excitatory synaptic input strengths (Figures $3 \mathbf{F}, \mathbf{G}$ ). As the level of ongoing background input increased, relatively smaller weights were needed on the feed-forward inhibitory synapse for optimal transmission.

One might imagine that the reduced weights needed for optimal transmission in the high noise case could arise from either the mean increase in excitation in the noise case, the variation provided by the noise, or both. To test the dependence on the overall mean excitation, we simulated a family of noiseless models where the reversal potential of the leak current was adjusted, effectively reducing the threshold and increasing the cell's excitability (Figures $\mathbf{3 H}, \mathbf{I}$ ). The optimal weight of the inhibitory input did not change with the change in excitability. This result suggests that the increase in the mean excitability in the noise cases did not influence the optimal value of $\alpha$, but, instead, that it was the variation in the input that needed to be balanced with less inhibition.

\section{Influence of Inhibitory Delay on Feed-Forward Transmission}

Another factor that should have a large influence on the temporal capabilities of feed-forward synapses is the delay between the feed-forward excitatory and inhibitory inputs. To examine this dependency, we varied the delay between excitation and inhibition systematically for a wide range of input modulation frequencies (Figure 4). Shorter delays allowed transmission at much higher temporal frequencies, and a delay of $20 \mathrm{~ms}$ produced a transmission profile that was almost as slow as excitatory inputs alone. Therefore, a short delay between feed-forward excitation and feed-forward inhibition is necessary to achieve the high temporal frequency transmission characteristics reported here.

\section{Paired Feed-Forward Excitatory and Inhibitory Inputs Act as a Clock or Trigger That can Organize Feed-Forward Computation Across Layers or Networks}

Neural circuits differ from conventional digital electronic circuits in that there is no external clock signal that tells neurons when they should begin evaluating their inputs (von Neumann, 1963). In digital electronics, a gate only updates its output when the clock signal indicates that it should re-scan its inputs. One classic idea suggests that neural circuits operate by attractor dynamics and therefore do not have a need for clocks (Hopfield and Tank, 1986; Miller, 2016). However, attractor dynamics take time to converge, but organisms are able to make extremely fast decisions in a few $100 \mathrm{~ms}$ post-stimulus (Thorpe et al., 1996; Sherwin et al., 2012). A feed-forward trigger would be useful for performing such fast computations. Here we examined whether paired feed-forward excitation and inhibition could provide temporal organization of their own input, in essence providing the feed-forward input values ("what to evaluate") and clock timing information ("when to compute") in a single mechanism.

We studied transmission across four cells in successive network levels in three different models. One might imagine that these cells represent the same receptive field location across these four network levels, so they have direct connections. However, each cell was also assumed to have local, ongoing excitatory input from its local network that was modeled as 50 noisy Poisson inputs that were not related to the feed-forward input. In one model, these four neurons were not connected at all, so that the baseline activity of these neurons could be 
studied as a control. In another model, these neurons were connected using FFE projections. These connections represent the simplest feed-forward cross-network computation that we could imagine, which is $\mathrm{y}=\mathrm{x}$. This computation was chosen because of its ease of evaluation, but these results should extend for any convergent feed-forward computation where the output is a function of the inputs at a particular time. Finally, we examined a model where cross-network transmission was provided by FFEI synapses across the four model network levels.

We evaluated how well the cells could transmit information forward to their subsequent levels, despite the ongoing local input that was unrelated to the feed-forward inputs. We assessed the temporal fidelity of the computation using the same frequency analysis that we performed in Figure 1. We provided an input firing rate that was modulated at a particular frequency that was varied and examined the Fourier coefficient (Figure 5B), overall activity (Figure 5C), and the normalized Fourier coefficients that indicated how specifically the output response followed the input frequency (Figure 5D). When FFE projections were used, the network level 4 output neuron did not efficaciously follow the input modulation frequency for frequencies greater than about $5 \mathrm{~Hz}$. However, when FFEI projections were used, the network level 4 output neuron followed the input very well at input modulation frequencies as high as $100 \mathrm{~Hz}$. This difference between FFE and FFEI was robust over a variety of feed-forward synaptic strengths (Figure 5E). Even when feed-forward firing rates were set so that the output neuron was driven at $20 \mathrm{~Hz}$ for $5 \mathrm{~Hz}$ input modulation, FFEI projections provided more than an 8-fold increase in transmission compared to FFE inputs modulated at $50 \mathrm{~Hz}$, and differences were more pronounced for higher synaptic strengths (Figure 5F). The $\frac{1}{2}$ cutoff frequency for the same network was more than 5 -fold greater (Figure 5G).

Interestingly, when FFE projections were used, the Fourier coefficient of the network level 4 output peaked several times at higher input frequencies $(300-1,000 \mathrm{~Hz})$. This effect can likely be credited to over-excitation of the network level 4 neuron, as seen in Figure 5A. Indeed, at most frequencies, the network level 4 neuron with FFE is likely firing at its maximum rate, due to the amplification of excitatory input over the circuit. As a result, high FC measurements at high input frequencies cannot necessarily be attributed to the circuit following the inputs, but may instead result from the physical features of the neuron allowing for maximal firing at these rates. In either case, the Fourier coefficient peaks at these high input frequencies do not stand out from the overall power of the output response, as shown in Figure 5D.

One may ask how much of these frequency responses could have arisen by chance due to the random activity that is provided to each network level. The fold increase in Fourier coefficient responses at the input modulation frequency is shown in Figure $\mathbf{5 H}$. Even at the lowest synaptic strength tested, FFEI projections provided responses that were at least five times those of the noise-only network, and this difference greatly increased when input strength was increased.

So far, we have only investigated how inputs of fixed modulation frequency are transmitted over multiple network levels. While this addresses how different network levels can exhibit synchronized activity, it does not directly confront how these networks can adapt to changing inputs. We, therefore, constructed a rectified sinusoidal Poisson input with a changing modulation frequency over time (Figure 5I). After changing the input modulation frequency, we held the input modulation frequency constant for $2 \mathrm{~s}$ in order to produce a reliable estimate of the Fourier coefficients. We then provided this input to the FFE and FFEI models and examined the normalized FC spectrum over time (Figures 5J,K), which measured how closely the network level 4 output followed a range of frequencies over the course of the input. With FFE projections, the network level 4 output only noticeably followed the stepping input at low frequencies $(5-10 \mathrm{~Hz})$. Meanwhile, with FFEI projections, the network level 4 output followed the entire stepping input fairly well. Furthermore, the network level 4 output did not exhibit significant residual power at frequencies represented earlier in the input, suggesting that the FFEI model was capable of quickly adapting to the changing input frequency. To more closely examine the speed at which the FFEI model transitions between inputs of different modulation frequencies, we provided the model with a Poisson input that increased from $F=10 \mathrm{~Hz}$ to $F=50 \mathrm{~Hz}$ at time $=1 \mathrm{~s}$, and computed the FC spectrum of the network level 4 output over smaller time intervals $(0.2 \mathrm{~s}$; Figure 5L). With this increased temporal resolution, the FFEI model rapidly adapts to an increase in the input frequency.

Taken together, these results indicate that FFEI projections organize multi-level, hierarchical computations that are also being driven by ongoing incidental activity. Further, FFEI projections allow these computations to occur with high fidelity even at high temporal frequencies. In this way, FFEI projections serve to deliver both a signal as well as the timing signal necessary to organize feed-forward computation across layers of processing.

\section{Paired Inputs of Opposite Signs can Organize Computation in Neuromorphic Circuits}

We have already noted that paired feed-forward excitation with slightly delayed inhibition is a common circuit motif in the brain, but the concept can be used more generally for computations with either sign. Although most neuronal circuits of which we are aware follow the excitatory-before-inhibitory configuration, there are some places, such as chandelier cells in the somatosensory cortex (Zhu et al., 2004), where inhibitory input precedes excitatory input. The paired E/I or I/E motifs can be used in artificial circuits to organize feed-forward computations at high speeds that exceed the time constant of integration of the individual elements.

We demonstrate the use of mixed feed-forward inputs in a model LIF circuit that performs the exclusive-or (XOR) 


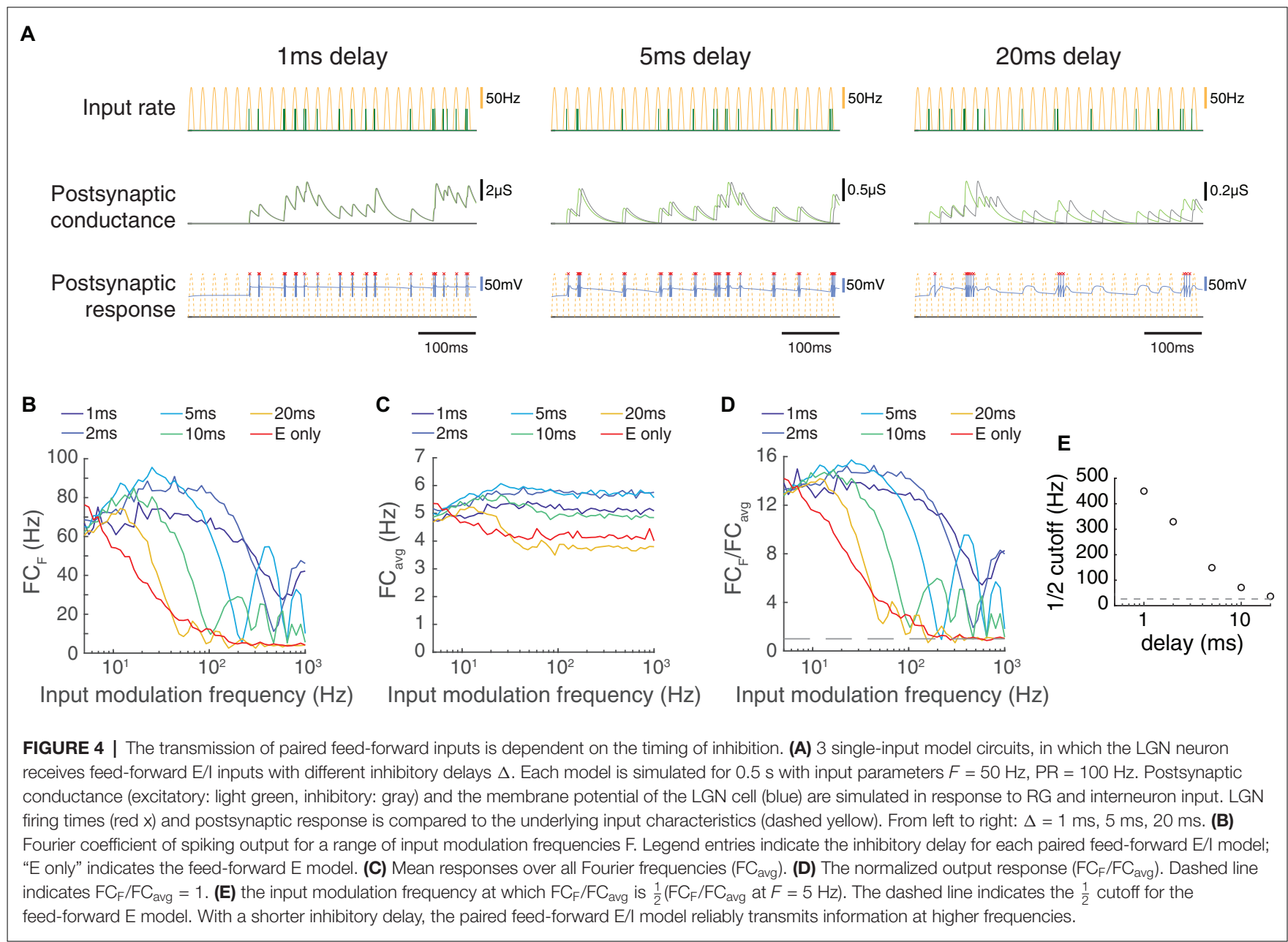

computation of two inputs (Figure 6). In this computation, the output neuron should exhibit a response if either of the two inputs is positive, but it should be silent if there is no input or if both inputs are active. To transmit a positive postsynaptic signal, we used FFEI synapses employing excitation that was followed by identical but delayed inhibition (Figure 6A). To transmit a negative signal, we employed inhibition that was followed by identical but delayed excitation. Input weights for cells in the first layer were set so that one input was positive and the other input was negative. Further, the input with a weight that was positive in the first cell had a negative weight in the second cell. The neuron in the second layer responded if either of the cells in the first layer responded.

Once again, we provided input at different modulation frequencies, although we shifted the two inputs so that they were $180^{\circ}$ out of phase (Figure 6A). Therefore, the XOR output should be produced at twice the input frequency (the cell should respond strongly when either input is at its positive phase, and weakly or not at all when the inputs are turned off). Once again, we found that the circuit with mixed FFEI projections could follow the XOR computation over high frequencies, while a comparison FFE circuit fell off rapidly with frequency, for both the raw response (Figure 6B) and for the fraction of the total response (Figure 6C) that was at the stimulus frequency (Figure 6D). We compared another input case (with the two inputs shifted by $90^{\circ}$ ) in Supplementary Figure 2.

We conclude that the concept of mirroring a feed-forward input with a delayed negative copy can organize high frequency computations across multi-layer neuromorphic circuits in much the same way that a clock organizes computations across digital electronic circuits.

\section{In Principle, AMPA-Only Currents Allow High Frequency Computations}

Most excitatory synapses in the mammalian brain employ a combination of the AMPA and NMDA receptors. AMPA receptors respond rapidly and briefly to neurotransmitters, while NMDA receptors remain open for longer periods of time (50-150 ms). However, there are a few instances of excitatory synapses that primarily involve AMPA receptors. One example is the calyx of Held synapse in the mature mammalian auditory brain stem; notably, this region often processes high frequency temporal information in the form of auditory input (Nakamura and Cramer, 2011). This raises the question of whether neural circuits could, in principle, achieve the same fast computation as 

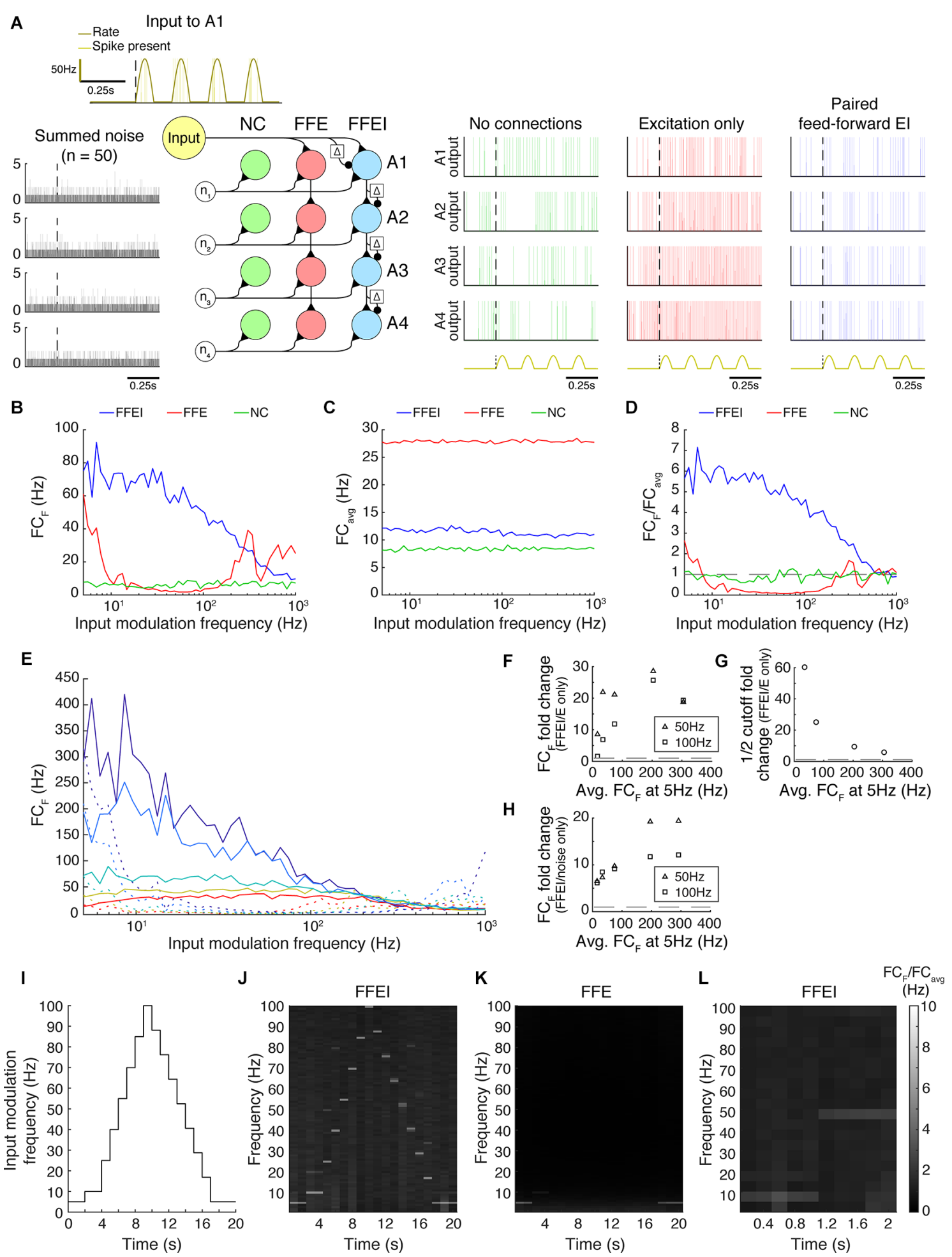

FIGURE 5 | Paired feed-forward E/l input allows time-locked high frequency computation across multiple brain layers or networks in the absence of an explicit external clock. (A) Three model circuits exhibiting different forms of $y=x$ transmission over four network levels (A1-A4): no connections between networks (NC), feed-forward E-only (FFE) connections, and paired feed-forward E/I (FFEI) connections. Each circuit receives the same Poisson input (yellow) at A1, with input firing rate a rectified sine wave of frequency F and amplitude PR (dark yellow). The circuits also receive 50 "internal" noisy Poisson excitatory inputs at A1-A4 (gray, left). Simulations (NC: green, FFE: red, FFEl: blue) with input parameters $F=5 \mathrm{~Hz}, \mathrm{PR}=100 \mathrm{~Hz}$ shown (repeated at bottom). (B) For a range of input modulation frequencies $F$, the Fourier coefficient of the $A 4$ output at $F\left(F C_{F}\right)$ was substantially higher for FFEl connections than for FFE connections. (C) Mean Fourier

(Continued) 


\section{FIGURE 5 | Continued}

coefficient of the $\mathrm{A} 4$ output over all frequencies ( $F \mathrm{C}_{\text {avg }}$ ). (D) Normalized Fourier output for a range of input modulation frequencies $F$. Dashed line indicates $\mathrm{FC}_{\mathrm{F}} / \mathrm{FC}_{\text {avg }}=1$. FFEl allows the network levels to follow computations at higher frequencies than FFE. (E) Same as (C), except that synaptic strength $\mathrm{P}_{\max }$ was adjusted to produce a range of Fourier coefficient values at an input modulation frequency at $5 \mathrm{~Hz}$ (weak to strong: red to dark blue). Solid lines indicate the feed-forward E/I model, and dashed lines indicate the feed-forward $E$ model. (F) Summary data from $(\mathbf{E})$, showing fold change advantage of FFEl over FFE at $F=50 \mathrm{~Hz}$ (triangles) and $F=100 \mathrm{~Hz}$ (squares) for various synaptic strengths ( $F C_{F}$ at $5 \mathrm{~Hz}$ shown on the $x$ axis). The dashed line indicates 1. (G) Same, but fold change in the $\frac{1}{2}$ cutoff shown. The $\frac{1}{2}$ cutoff fold change could not be measured for the weakest FFEI/FFE pair in (E), as the paired feed-forward E/I model does not have a $\frac{1}{2}$ cutoff within the range of considered $F$ values. (H) For each paired feed-forward $E / /$ model shown in $(\mathbf{E})$, the $\mathrm{FC}_{\mathrm{F}}$ fold change with respect to the model with no connections (noise only) is shown. The dashed line indicates a fold change of 1. (I) Over a $20 \mathrm{~s}$ trial, a rectified sinusoidal Poisson input with a changing input modulation frequency was provided to the circuits exhibiting FFE and FFEI connections. (J) The normalized Fourier spectrum of the A4 output with FFEl connections, averaged over $1 \mathrm{~s}$ bins. Spectrum is averaged over 50 trials. (K) Same as (J), but with FFE connections, which cannot follow high frequencies. (L) The FFEl circuit was provided with a rectified sinusoidal Poisson input with an input modulation frequency that was stepped from $10 \mathrm{~Hz}$ to $50 \mathrm{~Hz}$ at $t=1 \mathrm{~s}$. The Fourier spectrum of the A4 output is averaged over $0.2 \mathrm{~s}$ bins, showing a rapid transition of the output firing.

FFEI by using feed-forward inputs that were comprised entirely of fast AMPA channels (see also Wolf et al., 2005).

To address this question, we compared feed-forward transmission in synapses that were comprised of AMPA receptors only, AMPA and NMDA receptors only (like FFE), and AMPA, NMDA, and GABA receptors (like FFEI) in Figure 7 . We again provided rectified sinusoidal input at an input modulation frequency, and examined the Fourier coefficient of the response at the input frequency (Figure 7B), the average response across all frequencies (Figure $7 \mathbf{C}$ ), and the normalized output frequency (Figure 7D). Unlike in previous simulations with feed-forward excitatory transmission alone, accurate high frequency transmission was possible when only fast-closing AMPA channels were present. However, when slow-closing NMDA channels were added, the excitatory conductance and the spiking response became blurred, leading to poor transmission of high frequency information. High frequency transmission was restored by inhibition via GABA channels.

To investigate the effects of local background activity on the ability of the synapses to follow high frequency inputs, we provided 50 noisy Poisson inputs, modeled as in Figure 5, and examined the output response (Figures $7 \mathbf{E}-\mathbf{G}$ ). In the presence of excitatory noise, the FFEI synapse overall followed rectified sinusoidal inputs more effectively than the AMPA-only feed-forward synapse. These observations can be attributed to the overall output power, which was somewhat higher for the AMPA-only feed-forward synapse than for the FFEI synapse (Figure 7F).

These results suggest that AMPA-only feed-forward synapses provide some of the benefits of FFEI with respect to high frequency computation. However, FFEI synapses are more effective than AMPA-only feed-forward synapses when noisy inputs are also provided, suggesting that inhibitory GABA channels may play a role in filtering out excitatory noise and thus overriding ongoing computations.

\section{High Frequency Transmission in a Triad Synapse Model With Synaptic Depression}

Up to now, we have not considered an important feature of synaptic transmission in real triad synapses in the LGN: short-term dynamics (Abbott et al., 1997; Tsodyks and Markram, 1997; Varela et al., 1997; Carandini et al., 2002; Swadlow et al., 2002; Boudreau and Ferster, 2005; Higley and Contreras, 2006). The retino-geniculate excitatory and inhibitory synapses, as measured ex vivo in mice, both exhibit substantial short-term depression (Eysel, 1976; Koch, 1985; Chen and Regehr, 1999; Blitz and Regehr, 2003, 2005). We developed a depression model for excitatory and inhibitory synapses based on experimental data from mice (Chen and Regehr, 1999; Blitz and Regehr, 2003, 2005) using depression equations from Varela et al. (1997), and added AMPA, NMDA, GABA $A$, and $\mathrm{GABA}_{\mathrm{B}}$ currents (Supplementary Figure 3). NMDA and $\mathrm{GABA}_{\mathrm{B}}$ currents have long time courses, and it is necessary to have compatible time courses for the paired feed-forward input to allow high frequency transmission.

Adding depression dynamics also meant that we needed to consider the state of the synapses at the beginning of the simulation. In vivo in the cat, retinal ganglion cells exhibit relatively high spontaneous firing rates of tens of spikes/sec (Kuffler, 1953), so it is unlikely that retinal ganglion cell synapses would commonly be in a completely undepressed state; the neuron would need to be shown a stimulus that suppresses its firing, such as showing an ON spot to an OFF-center neuron, to achieve such a state. Therefore, although we began our simulations with the synapses in a completely undepressed state, we did not analyze the output until after $1 \mathrm{~s}$ had elapsed.

Representative simulations for synapses with AMPA + NMDA (FEI) and AMPA, NMDA, GABA A and GABA (FFEI) receptors are shown in Figures $\mathbf{8 A}, \mathbf{B}$. When the simulations begin, there is strong excitatory transmission, but excitation and inhibition become more balanced as the simulation runs. Synaptic weights were chosen for excitatory and inhibitory synapses so as to allow moderate firing in the FFEI case after $1 \mathrm{~s}$ of simulation; inhibitory inputs were removed for the FFE case, and the excitatory weights were adjusted so that the firing rate for inputs at $5 \mathrm{~Hz}$ modulation exhibited $70 \mathrm{~Hz}$ after $1 \mathrm{~s}$ of simulation, matching the FFEI simulations. With synaptic depression, transmission (Figures $\mathbf{8 C}-\mathbf{E}$ ) is improved for FFEI synapses compared to the simulations without synaptic depression, consistent with previous theoretical analyses (Abbott et al., 1997). However, transmission at higher temporal frequencies is better with the FFEI synaptic arrangement as compared to FEI.

We modulated the input rate sinusoidally from 0 to 50 spikes/s, a reduction from the 0 to 100 spikes/s we used in the prior examples. Blitz and Regehr (2005) noted that high frequency spike trains $(50-100 \mathrm{~Hz})$ caused such substantial depression that the inhibitory synapse effectively stopped transmitting. Here, it is important to note that we are modulating the firing rate probability of the input sinusoidally, 


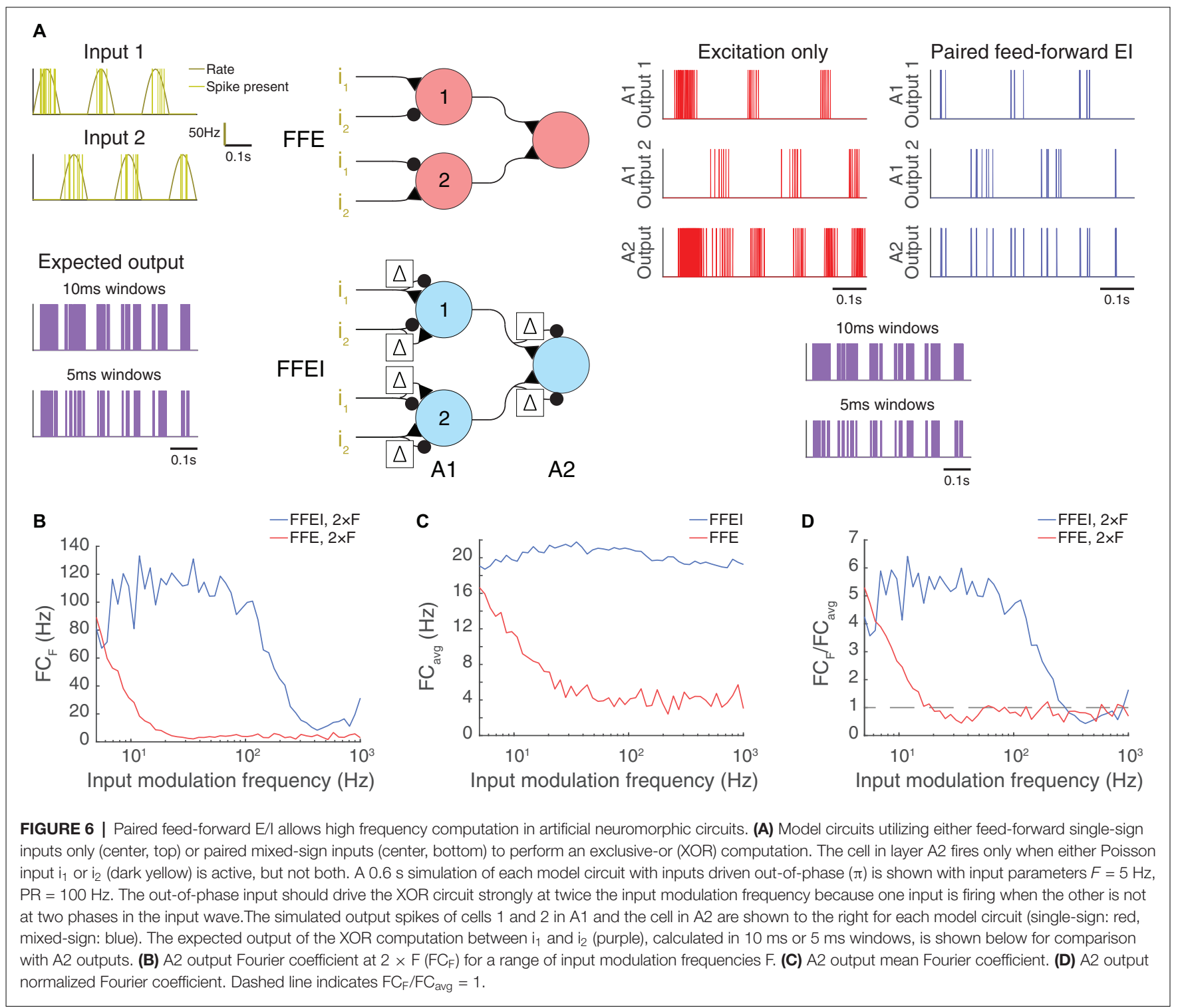

and a $50 \mathrm{~Hz}$ peak firing rate probability modulated sinusoidally will rarely result in spikes that have an inter-spike-interval of $20 \mathrm{~ms}(1 / 50 \mathrm{~Hz})$. Visual firing rates are typically quantified in a trial-averaged fashion and expressed as the probability of firing in a small time bin, and we are generating spikes according to such a process. At sustained high frequency input rates $>50 \mathrm{~Hz}$, it is likely that transmission of both excitation and especially inhibition would be very reduced, although it is unclear how common this situation of sustained high input rates would be in vivo, or if synaptic depression is altered in vivo or across species.

\section{Transmission Is Limited by Inhibitory Neuron Properties When FFEI Is Provided at the Circuit Level}

While the FFEI motif is sometimes found in inputs onto individual neurons, such as in the retinogeniculate triad synapse, the FFEI motif is also commonly found in inputs to circuits such as in the hippocampus (Buzsaki, 1984; Pouille and Scanziani, 2001; Bhatia et al., 2019) or the cerebral cortex, both in inputs from the thalamus (Agmon and Connors, 1991; Swadlow, 2003) and interareal projections (Yang et al., 2013). In these networks, excitatory projections from other areas make connections with separate populations of excitatory and inhibitory neurons, and the targeted inhibitory neurons provide rapid but slightly delayed inhibition onto the recipient excitatory neurons. Further, the excitatory and inhibitory neurons typically receive converging inputs from many cells, so that in principle the excitatory and inhibitory inputs to the local circuit may not be entirely matched. That is, the strong 1-to-1 relationship between each feed-forward excitatory postsynaptic potential and each feed-forward inhibitory postsynaptic potential that is found at the triad synapse is not present in circuit-level projections; instead, 


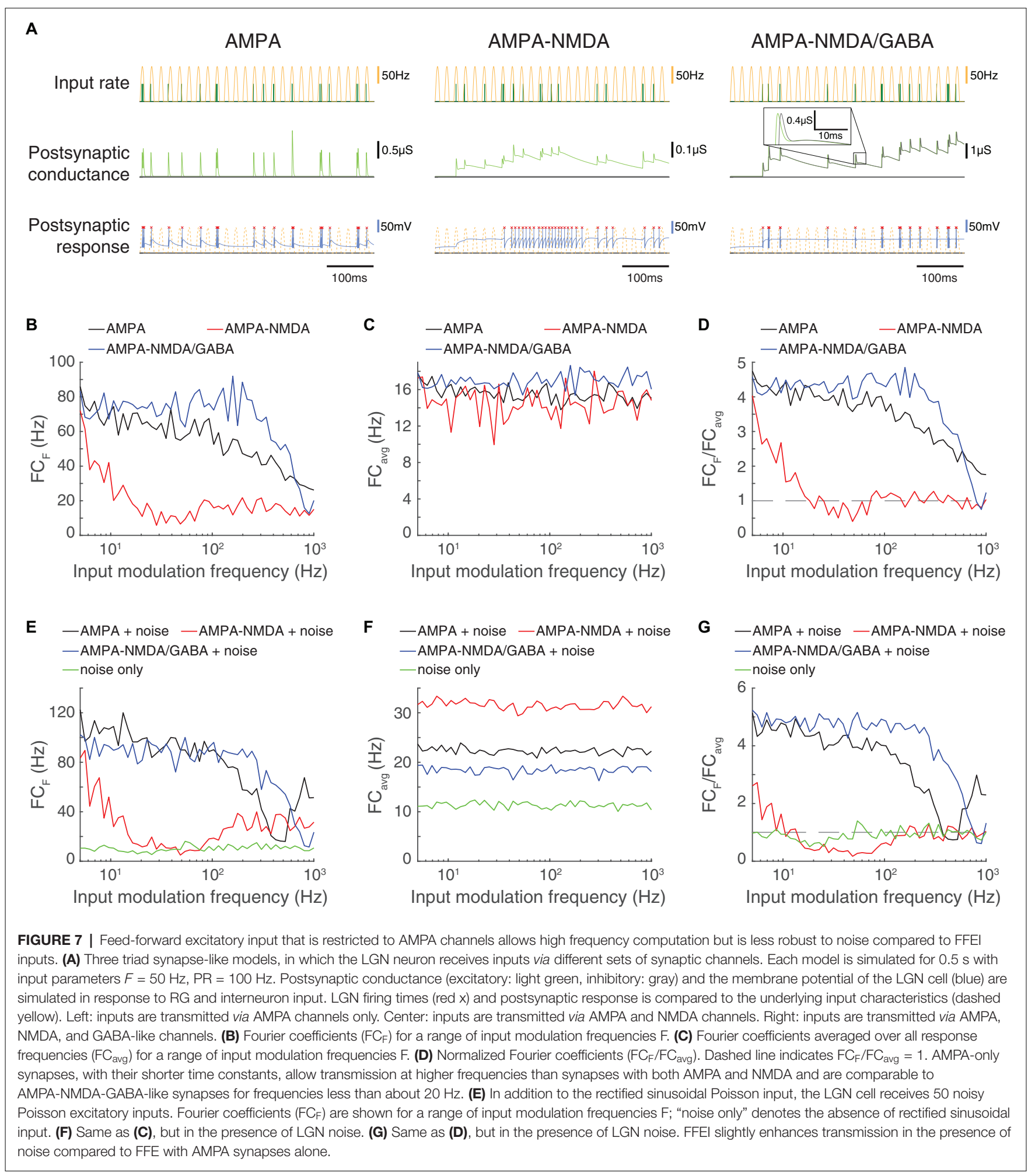

feed-forward excitation and inhibition are more loosely related.

Before we begin studying how FFEI can impact temporal computations at the circuit level, it is worth pausing to consider whether all cortical circuits might take advantage of any properties that we uncover. In the thalamus, it is clear that visual, somatosensory, and auditory neurons can follow sensory inputs at high frequencies. However, in visual (Hawken et al., 1996), somatosensory (Chung et al., 2002), and auditory cortex (Creutzfeldt et al., 1980; 
A
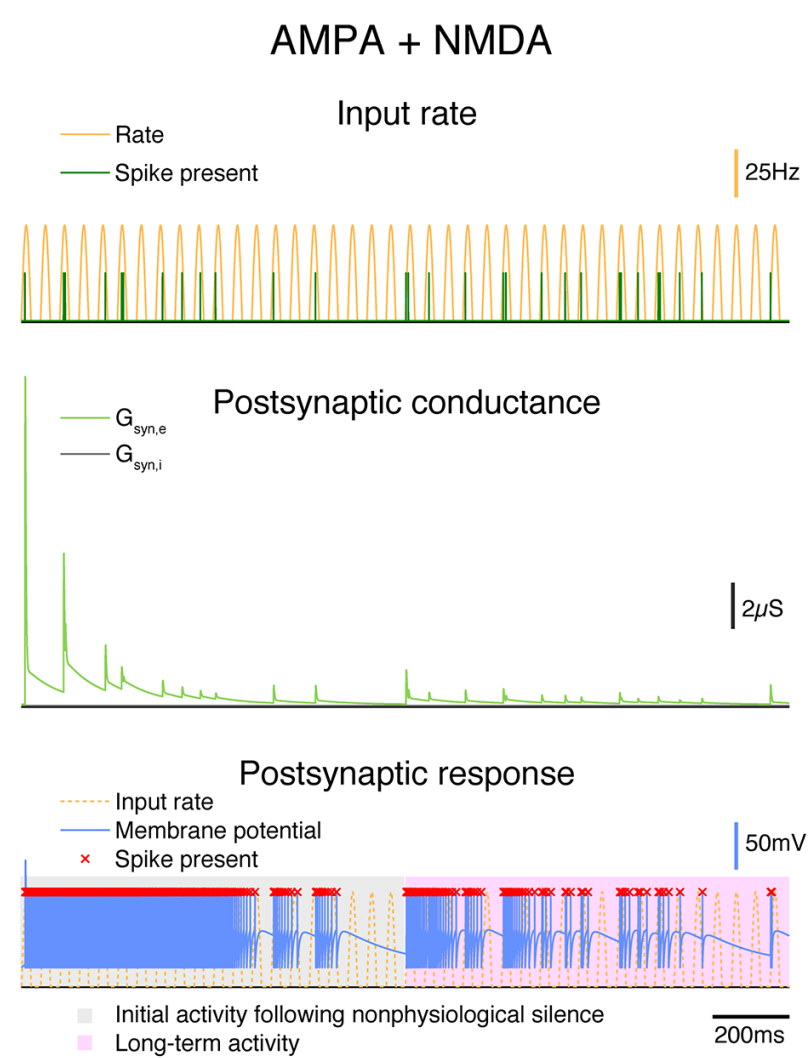

B

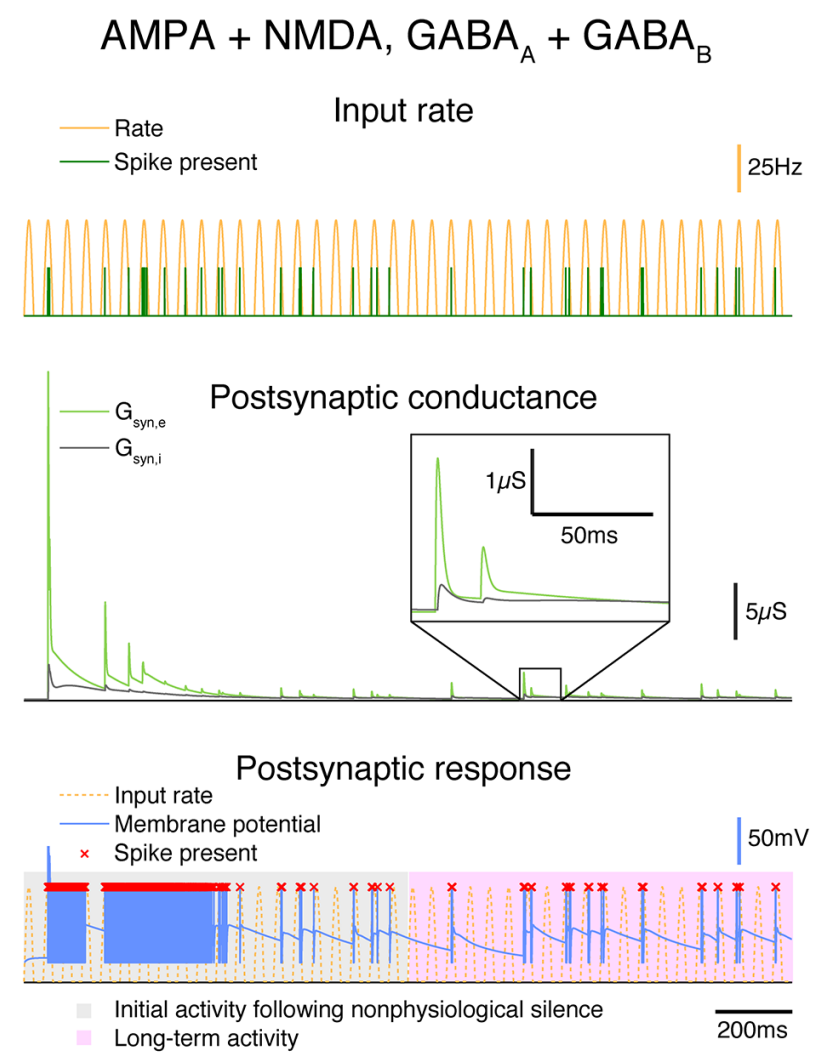

C

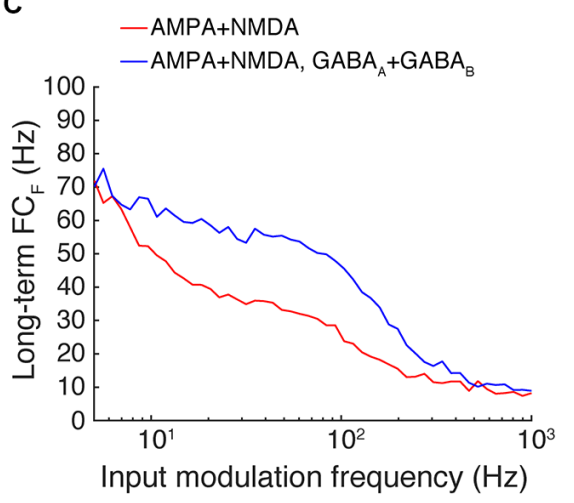

D

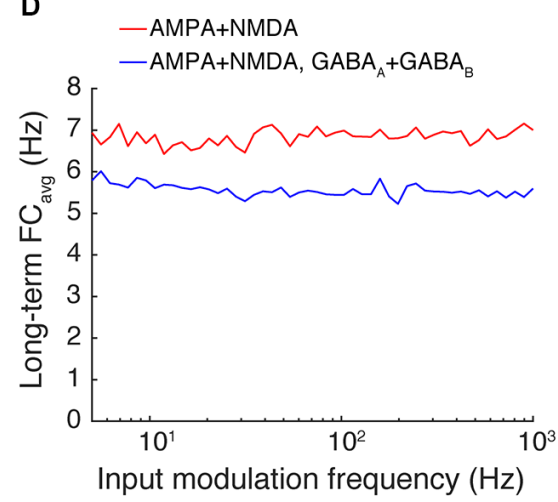

E

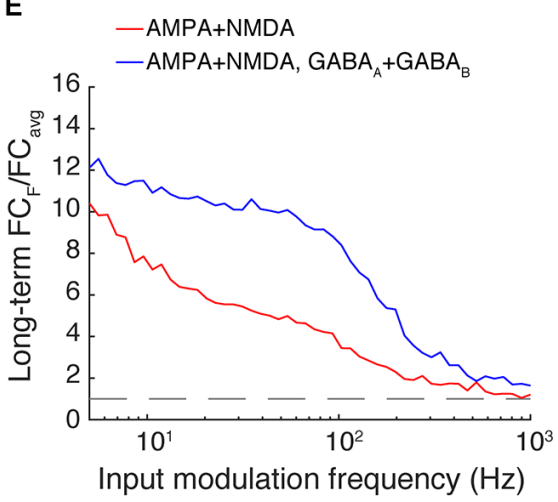

FIGURE 8 | In triad synapse models exhibiting synaptic depression, paired feed-forward E/l enhances high frequency transmission. (A,B) Two triad synapse models, in which the LGN neuron receives inputs via different sets of channels exhibiting synaptic depression. Each model is simulated for $2 \mathrm{~s}$ with input parameters $F=20 \mathrm{~Hz}, \mathrm{PR}=50 \mathrm{~Hz}$. Postsynaptic conductance (excitatory: light green, inhibitory: gray) and the membrane potential of the LGN cell (blue) are simulated in response to $R G$ and interneuron input. LGN firing times (red $\mathrm{x}$ ) and postsynaptic response is compared to the underlying input characteristics (dashed yellow). The output response is divided into 2 components over time: a $1 \mathrm{~s}$ period of initial activity following nonphysiological silence (gray highlight) and the subsequent long-term activity (pink highlight). (A) Inputs are transmitted via AMPA and NMDA channels only. (B) Inputs are transmitted via AMPA, NMDA, GABA A and GABA $_{B}$ channels. (C) Fourier coefficients $\left(F_{F}\right)$ for a range of input modulation frequencies $\mathrm{F}$. Models are simulated for $5 \mathrm{~s}$, and Fourier coefficients are computed $1 \mathrm{~s}$ after the start of each simulation to examine the long-term activity. (D) Fourier coefficients averaged over all response frequencies (FCavg) for a range of input modulation frequencies F. (E) Normalized Fourier coefficients $\left(F C_{F} / F C_{a v g}\right.$ ). Dashed line indicates $F C_{F} / F C_{a v g}=1$. With synaptic depression, AMPA-NMDA-GABA synapses allow for transmission at higher frequencies than synapses exhibiting excitation alone.

Wehr and Zador, 2005), cortical neurons primarily respond to stimulation at much lower stimulus repetition frequencies $(<20 \mathrm{~Hz})$ than do thalamic neurons. That is, the primary sensory cortex does not seem to segment responses to sensory stimulation at frequencies much higher than $20 \mathrm{~Hz}$. However, in both sensory and higher cortical areas and in 
the hippocampal formation, $40 \mathrm{~Hz}$ activity (gamma activity) is prominent (Fries et al., 2007) and can be induced by driving feed-forward interneurons (Cardin et al., 2009). It has been suggested that in hippocampal circuits, each gamma cycle might encode representations of individual items (Lisman and Idiart, 1995; Fries et al., 2007; Lisman and Jensen, 2013), and $40 \mathrm{~Hz}$ sensory stimulation has been reported to be protective against neurodegeneration (Iaccarino et al., 2016). So we feel it is worth considering whether circuit-level FFEI input can allow circuits to follow inputs at such high frequencies.

Here, we examined a model network with 100 feed-forward excitatory inputs that arrived at an LIF excitatory cell and an LIF inhibitory cell (Figure 9A). Following our previous methods, the firing rate of each input was modulated sinusoidally at an input modulation frequency. In this model, all inputs were driven at the same phase (Figure 9B). We examined the Fourier coefficient of the output responses at the input modulation frequency (Figure 9C), the average response across all frequencies (Figure 9D), and the ratio of these quantities (Figure 9E).

The circuit-level FFEI exhibited some similarities to and differences from the single FFEI input situation. One difference is that the circuit's I neuron receives excitatory input without a delayed inhibitory copy and acts just like a neuron receiving FFE input. Therefore, the frequency responses of the inhibitory neuron are the same as the E-only case in Figures $9 \mathrm{C}-\mathrm{E}$. If we look at these responses, we see that they fall off much like FFE input to the triad synapse: the response is much attenuated at about $25 \mathrm{~Hz}$, for example. At input modulation frequencies greater than this, the I neuron will not respond strongly to the excitatory input (Figure 9B, right), so the I neuron's contribution to the circuit is not present at high frequencies, and the circuit behaves like an FFE circuit. This means that the circuit-level FFEI projection has a much lower cut-off response frequency than a similarly-endowed triad-like FFEI projection.

We suspected that the membrane time constant of the inhibitory neuron would be a key parameter in determining the cut-off frequency for the circuit-level FFEI projection, so we compared responses in models where this parameter was varied. In this circuit network, the level of response output is very sensitive to the synaptic strengths of the inputs as well as the strength of the synapse from the inhibitory neuron onto the excitatory neuron. Because there were two non-linear ways to tune the responses to a particular input rate, we simply left the synaptic strengths unchanged while we altered the membrane time constant of the inhibitory neuron so that the models could be compared on the basis of that parameter.

In Figure 9F, we show the normalized Fourier coefficient for these models but with the data scaled so that the output response for an input modulation of $5 \mathrm{~Hz}$ is equal to 1 . Here it is apparent that the frequency response depends strongly on the inhibitory membrane time constant, with low values corresponding to high transmission at high input modulation frequencies. When the inhibitory time constant was $10 \mathrm{~ms}$, which is consistent with experimental studies of cortical interneurons (Economo and White, 2012), the $\frac{1}{2}$ frequency cut off was $40 \mathrm{~Hz}$, indicating that the excitatory cell could follow input responses very effectively up to that frequency (Figure 9G). This $\frac{1}{2}$ cutoff frequency was $70 \%$ greater than the $\frac{1}{2}$ cutoff frequency for a circuit with FFE projections alone (Figure $\mathbf{9 H}$ ).

We have just seen that the properties of the feed-forward inhibitory interneuron are critical to the temporal frequency limits of a circuit with paired feed-forward excitation and inhibition arriving via external excitatory input. In some circuits, the AMPA to NMDA current ratio is larger in feed-forward interneurons than in excitatory neurons (Wang and Gao, 2009). We have seen in Figure 8 that AMPA-only inputs allow high temporal frequency transmission, and we asked whether providing AMPA-only excitatory input to the feed-forward inhibitory neuron would increase the cut-off temporal frequency. In Figure 10, we reduced the synaptic time constant of the excitatory input to the inhibitory neuron from $20 \mathrm{~ms}$ to $1.6 \mathrm{~ms}$ to reflect an AMPA-dominated synapse. Indeed, the E neuron in the circuit can now follow much higher temporal frequencies, up to about $90 \mathrm{~Hz}$. These results indicate that the properties of the interneuron and its input are the key limiting factor to high temporal frequency transmission in these circuits.

\section{DISCUSSION}

We have shown that feed-forward excitation and delayed inhibition can enhance the transmission of high frequency temporal information through neural circuits and that this enhancement occurs for a broad range of synaptic and membrane parameters. Similar to the triad synapses in retinogeniculate projections, FFEI models with low input convergence and tightly paired E/I inputs exhibit robust temporal transmission over a wide range of frequencies up to $\sim 100 \mathrm{~Hz}$. Meanwhile, in circuits modeled after hippocampal and cortical projections, FFEI enhances high frequency transmission up to about $40-80 \mathrm{~Hz}$, despite high input convergence $(N=100)$ and a looser association between excitatory and inhibitory inputs. In circuit models of hierarchical networks, we found that FFEI projections reliably transmit high frequency signals and organize activity across network levels over time, suppressing the influence of ongoing activity and essentially acting as a feed-forward clock trigger. Thus, FFEI projections can act as a hybrid input, conveying both information about input value and imposing a clock-like trigger to initiate rapid computation.

\section{FFEI Allows Computation at High Temporal Frequencies That "Break the Limit" of the Membrane Time Constant}

Excitation with delayed inhibition produces a narrow time window during which spiking can occur. As a result, FFEI inputs can enhance the temporal fidelity of neural circuit outputs, as shown in experiments in hippocampal CA1 (Pouille and Scanziani, 2001; Bhatia et al., 2019) and at the retinogeniculate synapse (Blitz and Regehr, 2005). Given the high temporal precision of networks containing FFEI projections, 


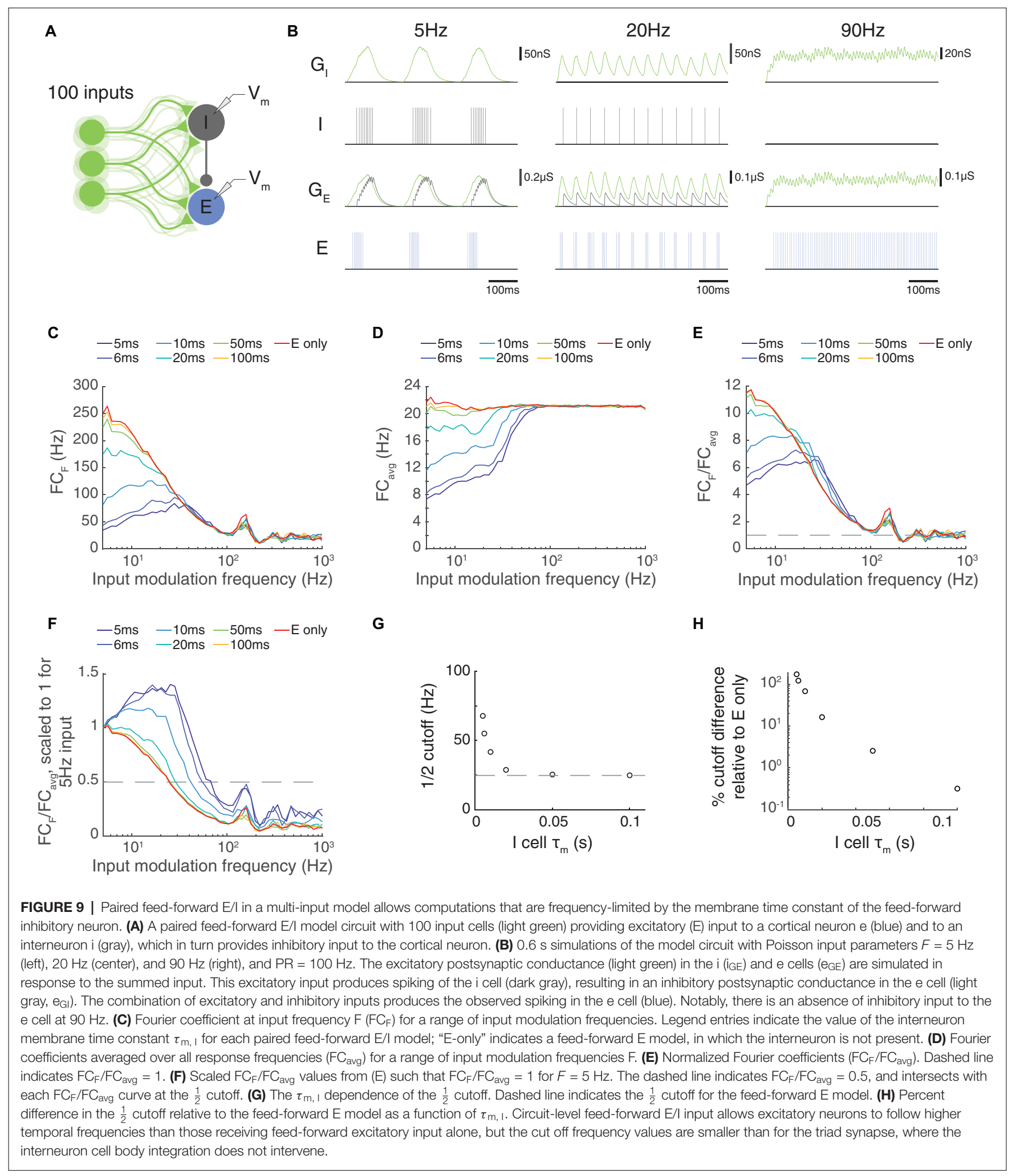

it follows that such networks would accurately transmit high frequency temporal information, as well. This effect can largely be attributed to the narrow spiking time window generated by paired E/I inputs, as previously described (Pouille and
Scanziani, 2001; Wehr and Zador, 2003; Blitz and Regehr, 2005; Mittmann et al., 2005; Higley and Contreras, 2006; Cardin, 2018). Here we show that the residual inhibition following each input spike does not suppress the cell for long, and the 


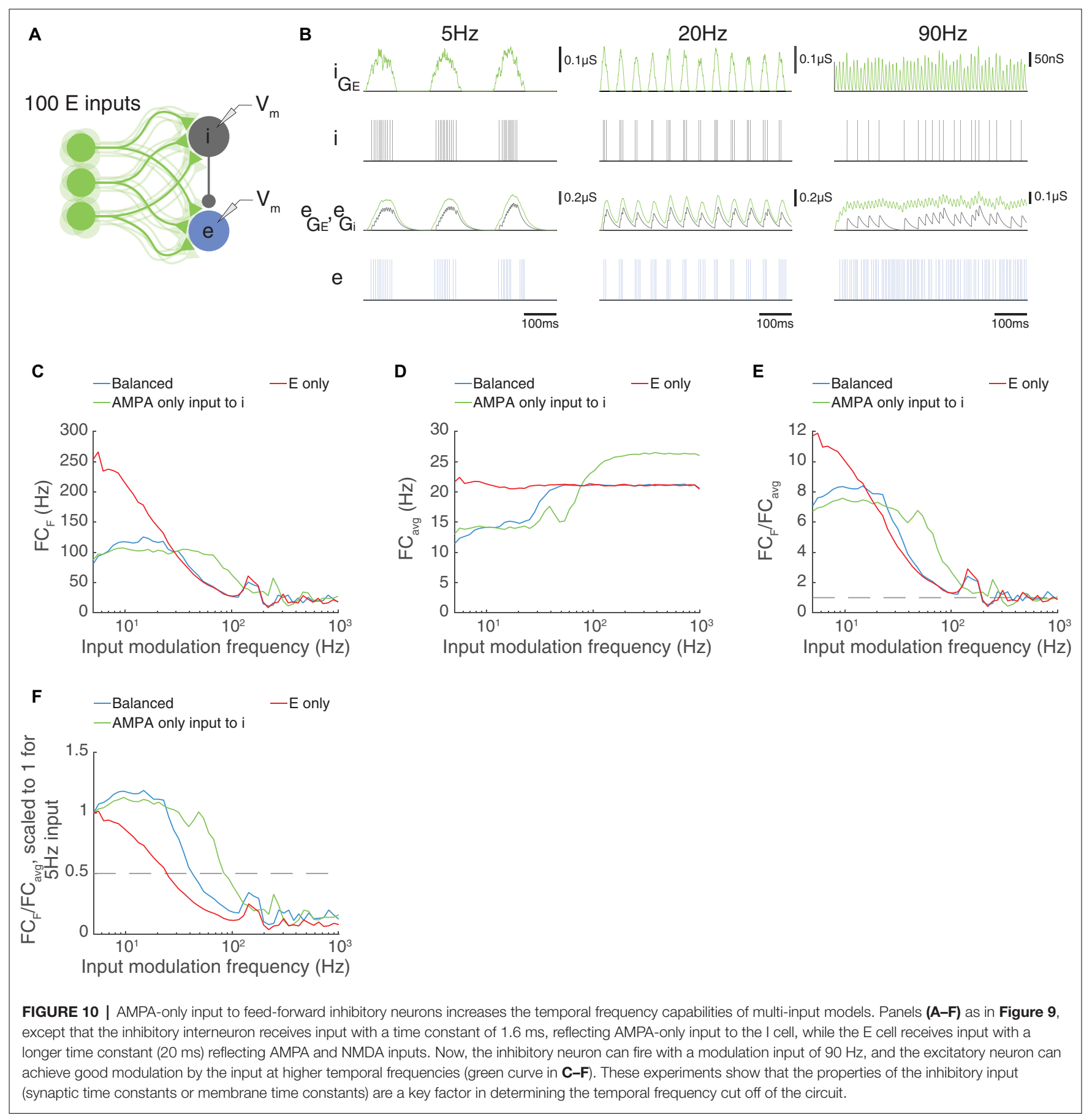

cell is quickly ready to respond to subsequent FFEI inputs (Figure 3A).

Networks that propagate signals with high temporal fidelity have been called synfire chains (Abeles, 1991). We have shown here that excitatory-only feed-forward networks can produce reliable computation only at relatively low temporal frequencies. Excitatory-only feed-forward networks can reliably and quickly transmit firing rate information if each area receives ongoing balanced excitatory and inhibitory internal noisy input (Shadlen and Newsome, 1998; Chance et al., 2002; van Rossum et al., 2002;
Murphy and Miller, 2009) or has detailed or loosely balanced strongly recurrent connections (Tsodyks and Sejnowski, 1995; van Vreeswijk and Sompolinsky, 1996, 1998; Hennequin et al., 2017) or balanced connectivity across cortical areas (Joglekar et al., 2018). Here we have shown that this transmission is possible with FFEI projections even if the random local input that a local area experiences or the internal connectivity of the network is not balanced: transmission at high temporal frequencies can be accomplished with balanced feed-forward input alone. 
It should be noted that some brain pathways use different strategies to transmit high frequency information. In the auditory brainstem, neurons respond rapidly to a transient input (Schnupp and Carr, 2009), which can be explained by the composition of the intrinsic ionic currents in the cell that allow them to act as "slope detectors" (Meng et al., 2012), although they do not respond tonically to constant current, as LGN neurons do (Suzuki and Rogawski, 1989).

The high transmission functions of FFEI could act in concert with another proposed function for the triad synapse: a state-dependent "veto" (Koch, 1985). Under this idea, the synapse would act as described here when operating in a feed-forward mode, but the inhibitory neuron could be activated independently in order to suppress feed-forward excitatory input to support functions such as reduced attention or internal processing. A related feed-forward excitatory/inhibitory gate model was demonstrated by Kremkow et al. (2010a). In recurrent, balanced cortical networks, inhibitory synaptic strengths (Vogels and Abbott, 2009) or the correlation between excitatory and inhibitory signals can be used to gate the propagation of tonic activity through downstream networks, and this can be combined with temporal gating (Kremkow et al., 2010a). Learning rules (Vogels et al., 2011; Kleberg et al., 2014) could tune inhibition for ideal propagation at high temporal frequencies (see Figure 3).

\section{FFEl as a Feed-Forward "Clock" Trigger Mechanism}

The absence of a synchronizing clock or trigger signal to organize the activity of neurons would seem to imply that neuronal circuits would be limited to performing computations that do not require such a trigger, such as attractor networks or integrators (Hopfield, 1982; Seung et al., 2000; Miller et al., 2003; Miller, 2016). FFEI acts much like a feed-forward synchronizing trigger that forces computation to occur within a short window (Buzsaki, 1984; Agmon and Connors, 1991; Swadlow and Gusev, 2000; Porter et al., 2001; Swadlow, 2003; Rock and Apicella, 2015; Bhatia et al., 2019).

Here we showed that FFEI when used in projections across brain areas or layers, can organize computation across these regions, even when these regions are experiencing ongoing local activity (Figure 5). Unlike a digital computer, where the clock trigger runs at a fixed rate, FFEI allows the trigger signal to be generated at any time (Figure 5I-K) and at many rates, up to $100 \mathrm{~Hz}$ for triad-like synapses and up to about $40-80 \mathrm{~Hz}$ for circuit-level FFEI inputs, depending upon the assumptions of the model. In low-level areas, these triggers would be generated when sensory input arrives, and computation could proceed at the speed of the sensory input. In higher-level areas, computation across two areas could proceed whenever the input area generates output and could underlie coordination of high frequency oscillations across areas (Lisman and Idiart, 1995; Lisman and Jensen, 2013).

The degree to which high frequency information actually travels throughout the brain varies depending upon area and channel and has been well studied in the visual system. In recordings that examined velocity tuning to single bar sweeps in both retina and LGN of $\mathrm{X}$ and $\mathrm{Y}$ cells in the cat, peak velocity tuning was slightly lower in LGN for $\mathrm{X}$ cells, but very comparable in retina and LGN for Y cells, with preferred velocities that ranged from about $20^{\circ} / \mathrm{s}$ to $100^{\circ} / \mathrm{s}$ (Frishman et al., 1983), indicating that spike timing information can be precisely transmitted. Consistent with this idea, retinal inputs activate LGN cells with high efficacy (Usrey et al., 1998), indicating that LGN neurons follow retinal ganglion inputs with high reliability.

Evidence from sensory cortex, however, suggests that the thalamocortical connection does not take full advantage of the potential FFEI speeds. There is a drop in peak temporal frequency tuning between LGN and cortex, with most LGN cells exhibiting strong firing for many frequencies, including those greater than $16 \mathrm{~Hz}$, while few cortical neurons exhibit strong firing at $16 \mathrm{~Hz}$ or greater (Hawken et al., 1996; Van Hooser et al., 2003, 2013; Heimel et al., 2005). If FFEI were the only factor, we would predict that transmission should be quite robust at $20 \mathrm{~Hz}$ (Figure 9), but the visual cortex attenuates these frequencies quite strongly. This drop also occurs in the somatosensory (Ahissar et al., 2001; Chung et al., 2002) and auditory systems (Creutzfeldt et al., 1980). Synaptic depression or other cortical circuit mechanisms may underlie this filtering (Chung et al., 2002; Swadlow, 2002; Boudreau and Ferster, 2005; Swadlow et al., 2005; Wehr and Zador, 2005).

Nevertheless, many cortical regions exhibit strong modulation and resonance at around $40 \mathrm{~Hz}$, also known as the gamma frequency (Fries et al., 2007; Cardin et al., 2009; Lisman and Jensen, 2013). If responses at $40 \mathrm{~Hz}$ in one area are to be intelligible in another cortical or hippocampal area, then it is necessary for these circuits to be able to follow inputs at these frequencies. Here we showed that circuit-level FFEI inputs can in principle allow such rapid communication.

\section{Predictions}

The FFEI models make a simple prediction: the absence of inhibition at cells receiving FFEI inputs should limit the ability of these cells to follow inputs at high temporal frequencies. An optogenetics approach could be used to control the activation of retinal ganglion cells (RGC) with high temporal precision, and the activity of LGN relay cells in response to RGC action potentials could be measured. A GABAzine infusion to block inhibition in the LGN should limit the ability of LGN relay cells to follow RGC inputs at high temporal frequencies. A similar experiment could be performed at thalamocortical slices in the somatosensory cortex (Agmon and Connors, 1991) in order to test the high-convergence regime. While we have noted that there is a high degree of synaptic depression in thalamocortical synapses (Chung et al., 2002; Higley and Contreras, 2006), the data from an experiment should exhibit a strong shift, as in Figure 9, from the FFEI dynamics towards E-only dynamics. One experiment that studied the propagation of signals through the somatosensory cortex in vivo found that spike timing transmission precision decreased at low firing rates (but not for high firing rates) when PV interneurons were silenced (Jang et al., 2020). 


\section{CONCLUSION}

Feed-forward pairing of excitatory and inhibitory connections provides several functions for neural circuits. It helps to linearize computations that are impacted by rectification in the retina (Werblin, 2010), LGN, and cortex (Hirsch et al., 2015). It sharpens stimulus tuning and spike timing (Pouille and Scanziani, 2001; Wehr and Zador, 2003; Blitz and Regehr, 2005; Mittmann et al., 2005; Higley and Contreras, 2006; Cardin, 2018), and allows neurons to respond to weak inputs without saturating for strong inputs (Pouille et al., 2009). In the hippocampus, the delay between excitation and inhibition shortens with stronger CA3 inputs, producing smaller changes in CA1 membrane potential (Bhatia et al., 2019). Therefore feed-forward excitation and inhibition allow neurons to accommodate a dynamic range of inputs before firing, providing a mechanism of gaining control of these circuits (Pouille et al., 2009; Bhatia et al., 2019). Here we add that it provides a dual signal-the value of its input on the one hand and a clock-like trigger on the other-that allows high frequency feed-forward computation that is synchronized across brain areas.

\section{DATA AVAILABILITY STATEMENT}

The datasets presented in this study can be found in online repositories. The names of the repository/repositories and accession number(s) can be found below: https://github.com/VH-Lab/vhlab-ffei-matlab.

\section{AUTHOR CONTRIBUTIONS}

AC performed modeling. AC and SVH designed the models. AC and SVH wrote the article. All authors contributed to the article and approved the submitted version.

\section{FUNDING}

This work was funded by NIH EY022122 (SVH) and NIH DA033463 (AC).

\section{REFERENCES}

Abbott, L. F. (1999). Lapicque's introduction of the integrate-and-fire model neuron (1907). Brain Res. Bull. 50, 303-304. doi: 10.1016/s03619230(99)00161-6

Abbott, L. F., Varela, J. A., Sen, K., and Nelson, S. B. (1997). Synaptic depression and cortical gain control. Science 275, 220-224. doi: 10.1126/science.275.5297. 221

Abeles, M. (1991). Corticonics: Neural Circuits of the Cerebral Cortex. Cambridge: Cambridge University Press.

Agmon, A., and Connors, B. W. (1991). Thalamocortical responses of mouse somatosensory (barrel) cortex in vitro. Neuroscience 41, 365-379. doi: 10.1016/0306-4522(91)90333-j

Ahissar, E., Sosnik, R., Bagdasarian, K., and Haidarliu, S. (2001). Temporal frequency of whisker movement. II. Laminar organization of cortical representations. J. Neurophysiol. 86, 354-367. doi: 10.1152/jn.2001.86.1.354

Bhatia, A., Moza, S., and Bhalla, U. S. (2019). Precise excitation-inhibition balance controls gain and timing in the hippocampus. elife 8:e43415. doi: $10.7554 /$ eLife.43415

\section{ACKNOWLEDGMENTS}

We thank Andrew Lipnick, whose undergraduate thesis on velocity tuning led us to consider feed-forward transmission, Paul Miller for useful suggestions, and members of the Van Hooser lab for comments.

\section{SUPPLEMENTARY MATERIALS}

The Supplementary Material for this article can be found online at: https://www.frontiersin.org/articles/10.3389/fncir.2021.8030 65/full\#supplementary-material.

SUPPLEMENTARY FIGURE 1 | High frequency transmission differs between excitation-only circuits and circuits exhibiting paired feed-forward excitation and delayed inhibition with long time constants. (A) $0.5 \mathrm{~s}$ simulation of a feed-forward E circuit receiving a single rectified sinusoidal Poisson input (yellow) with a peak rate (PR) of $100 \mathrm{~Hz}$ and an input modulation frequency $(\mathrm{F})$ of $40 \mathrm{~Hz}$, as in Figure 1A. (B) Same, but for a paired feed-forward E/I model circuit with $\tau_{\text {fall, } i}=50 \mathrm{~ms}$ instead of the baseline $20 \mathrm{~ms}$ shown in Figure 1A. Spiking activity in the postsynaptic neuron with $\tau_{\text {fall, } i}=50 \mathrm{~ms}$ is more prolonged than in the $20 \mathrm{~ms}$ case in Figure 1A, but the firing is still truncated by the inhibition, as compared to the no-inhibition case in panel (A)

SUPPLEMENTARY FIGURE 2 | Paired feed-forward E/I in an XOR circuit, with $\pi / 2$ phase-shifted inputs. (A) A simulation of each XOR model circuit described in Figure 6A is shown with inputs modeled as in Figure 6A, but with a $\pi / 2$ phase shift instead of a $\pi$ phase shift, which should result in output at the stimulus frequency $F$ (instead of $2 \times F$ when the phase shift is $\pi$ ). (B) For a range of input modulation frequencies $F$, the Fourier coefficient of the $A 2$ output at $F\left(F C_{F}\right)$ was averaged over 10 trials for the two XOR circuit models shown in (A). (C) For a range of input modulation frequencies $F$, the mean power of the $A 2$ output over all frequencies $\left(\mathrm{FC}_{\mathrm{avg}}\right)$. (D) For a range of input modulation frequencies $F$, the normalized Fourier coefficient of the A2 output is shown. Dashed line indicates $\mathrm{FC}_{\mathrm{F}} / \mathrm{FC}_{\mathrm{avg}}=1$.

SUPPLEMENTARY FIGURE 3 | Fitting depression at the retinogeniculate synapse. (A-C) The paired-pulse ratio for the AMPAR (A), NMDAR (B), and locked GABAR (C) components of the synaptic current was plotted over a range of interspike intervals. For each channel, raw data (black) obtained from voltage clamp experiments (Chen et al., 2002; Blitz and Regehr, 2005) were fitted to a depression model (see "Materials and Methods" section). Paired-pulse experiments were then simulated using the fitted depression model (red).

Bickford, M. E. (2019). Synaptic organization of the dorsal lateral geniculate nucleus. Eur. J. Neurosci. 49, 938-947. doi: 10.1111/ejn.13917

Blitz, D. M., and Regehr, W. G. (2003). Retinogeniculate synaptic properties controlling spike number and timing in relay neurons. J. Neurophysiol. 90, 2438-2450. doi: 10.1152/jn.00562.2003

Blitz, D. M., and Regehr, W. G. (2005). Timing and specificity of feed-forward inhibition within the LGN. Neuron 45, 917-928. doi: 10.1016/j.neuron.2005. 01.033

Boudreau, C. E., and Ferster, D. (2005). Short-term depression in thalamocortical synapses of cat primary visual cortex. J. Neurosci. 25, 7179-7190. doi: 10.1523/JNEUROSCI.1445-05.2005

Buzsaki, G. (1984). Feed-forward inhibition in the hippocampal formation. Prog. Neurobiol. 22, 131-153. doi: 10.1016/0301-0082(84)90023-6

Carandini, M., Heeger, D. J., and Senn, W. (2002). A synaptic explanation of suppression in visual cortex. J. Neurosci. 22, 10053-10065. doi: 10.1523/JNEUROSCI.22-22-10053.2002

Cardin, J. A. (2018). Inhibitory interneurons regulate temporal precision and correlations in cortical circuits. Trends Neurosci. 41, 689-700. doi: 10.1016/j. tins.2018.07.015 
Cardin, J. A., Carlen, M., Meletis, K., Knoblich, U., Zhang, F., Deisseroth, K., et al. (2009). Driving fast-spiking cells induces gamma rhythm and controls sensory responses. Nature 459, 663-667. doi: 10.1038/nature08002

Chance, F. S., Abbott, L. F., and Reyes, A. D. (2002). Gain modulation from background synaptic input. Neuron 35, 773-782. doi: 10.1016/s08966273(02)00820-6

Chen, C., Blitz, D. M., and Regehr, W. G. (2002). Contributions of receptor desensitization and saturation to plasticity at the retinogeniculate synapse. Neuron 33, 779-788. doi: 10.1016/s0896-6273(02)00611-6

Chen, C., and Regehr, W. G. (1999). Contributions of residual calcium to fast synaptic transmission. J. Neurosci. 19, 6257-6266. doi: 10.1523/JNEUROSCI. 19-15-06257.1999

Chen, C., and Regehr, W. G. (2000). Developmental remodeling of the retinogeniculate synapse. Neuron 28, 955-966. doi: 10.1016/s08966273(00)00166-5

Chung, S., Li, X., and Nelson, S. B. (2002). Short-term depression at thalamocortical synapses contributes to rapid adaptation of cortical sensory responses in vivo. Neuron 34, 437-446. doi: 10.1016/s0896-6273(02)00659-1

Cox, C. L., Zhou, Q., and Sherman, S. M. (1998). Glutamate locally activates dendritic outputs of thalamic interneurons. Nature 394, 478-482. doi: $10.1038 / 28855$

Crawford, F. S. (1968). Waves (Berekely Physics Course, Vol. 3). New York, NY: McGraw-Hill.

Creutzfeldt, O., Hellweg, F. C., and Schreiner, C. (1980). Thalamocortical transformation of responses to complex auditory stimuli. Exp. Brain Res. 39, 87-104. doi: 10.1007/BF00237072

Dayan, P., and Abbott, L. F. (2001). Theoretical Neuroscience. Cambridge, MA: MIT Press.

Destexhe, A., and Sejnowski, T. J. (1995). G protein activation kinetics and spillover of gamma-aminobutyric acid may account for differences between inhibitory responses in the hippocampus and thalamus. Proc. Natl. Acad. Sci. U S A 92, 9515-9519. doi: 10.1073/pnas.92.21.9515

Economo, M. N., and White, J. A. (2012). Membrane properties and the balance between excitation and inhibition control gamma-frequency oscillations arising from feedback inhibition. PLoS Comput. Biol. 8:e1002354. doi: 10.1371/journal.pcbi.1002354

Eysel, U. T. (1976). Quantitative studies of intracellular postsynaptic potentials in the lateral geniculate nucleus of the cat with respect to optic tract stimulus response latencies. Exp. Brain Res. 25, 469-486. doi: 10.1007/BF00239782

Fries, P., Nikolic, D., and Singer, W. (2007). The gamma cycle. Trends Neurosci. 30, 309-316. doi: 10.1016/j.tins.2007.05.005

Frishman, L. J., Freeman, A. W., Troy, J. B., Schweitzer-Tong, D. E., and Enroth-Cugell, C. (1987). Spatiotemporal frequency responses of cat retinal ganglion cells. J. Gen. Physiol. 89, 599-628. doi: 10.1085/jgp.89. 4.599

Frishman, L. J., Schweitzer-Tong, D. E., and Goldstein, E. B. (1983). Velocity tuning of cells in dorsal lateral geniculate nucleus and retina of the cat. J. Neurophysiol. 50, 1393-1414. doi: 10.1152/jn.1983.50. 6.1393

Gabernet, L., Jadhav, S. P., Feldman, D. E., Carandini, M., and Scanziani, M. (2005). Somatosensory integration controlled by dynamic thalamocortical feed-forward inhibition. Neuron 48, 315-327. doi: 10.1016/j.neuron.2005.09. 022

Hamos, J. E., Van Horn, S. C., Raczkowski, D., and Sherman, S. M. (1987). Synaptic circuits involving an individual retinogeniculate axon in the cat. J. Comp. Neurol. 259, 165-192. doi: 10.1002/cne.902590202

Hawken, M. J., Shapley, R. M., and Grosof, D. H. (1996). Temporalfrequency selectivity in monkey visual cortex. Vis. Neurosci. 13, 477-492. doi: 10.1017/s0952523800008154

Heimel, J. A., Van Hooser, S. D., and Nelson, S. B. (2005). Laminar organization of response properties in primary visual cortex of the gray squirrel (Sciurus carolinensis). J. Neurophysiol. 94, 3538-3554. doi: 10.1017/s0952523800008154

Hennequin, G., Agnes, E. J., and Vogels, T. P. (2017). Inhibitory plasticity: balance, control and codependence. Annu. Rev. Neurosci. 40, 557-579. doi: 10.1146/annurev-neuro-072116-031005

Higley, M. J., and Contreras, D. (2006). Balanced excitation and inhibition determine spike timing during frequency adaptation. J. Neurosci. 26, 448-457. doi: 10.1523/JNEUROSCI.3506-05.2006
Hirsch, J. A., Wang, X., Sommer, F. T., and Martinez, L. M. (2015). How inhibitory circuits in the thalamus serve vision. Annu. Rev. Neurosci. 38, 309-329. doi: 10.1146/annurev-neuro-071013-014229

Hopfield, J. J. (1982). Neural networks and physical systems with emergent collective computational abilities. Proc. Natl. Acad. Sci. U S A 79, 2554-2558. doi: 10.1073/pnas.79.8.2554

Hopfield, J. J., and Tank, D. W. (1986). Computing with neural circuits: a model. Science 233, 625-633. doi: 10.1126/science.3755256

Iaccarino, H. F., Singer, A. C., Martorell, A. J., Rudenko, A., Gao, F., Gillingham, T. Z., et al. (2016). Gamma frequency entrainment attenuates amyloid load and modifies microglia. Nature 540, 230-235. doi: 10.1038/nature20587

Jang, H. J., Chung, H., Rowland, J. M., Richards, B. A., Kohl, M. M., and Kwag, J. (2020). Distinct roles of parvalbumin and somatostatin interneurons in gating the synchronization of spike times in the neocortex. Sci. Adv. 6:eaay5333. doi: 10.1126/sciadv.aay5333

Joglekar, M. R., Mejias, J. F., Yang, G. R., and Wang, X. J. (2018). Inter-areal balanced amplification enhances signal propagation in a large-scale circuit model of the primate cortex. Neuron 98, 222-234.e8. doi: 10.1016/j.neuron. 2018.02.031

Kleberg, F. I., Fukai, T., and Gilson, M. (2014). Excitatory and inhibitory STDP jointly tune feedforward neural circuits to selectively propagate correlated spiking activity. Front. Comput. Neurosci. 8:53. doi: 10.3389/fncom.2014. 00053

Koch, C. (1985). Understanding the intrinsic circuitry of the cat's lateral geniculate nucleus: electrical properties of the spine-triad arrangement. Proc. R. Soc. Lond. B Biol. Sci. 225, 365-390. doi: 10.1098/rspb.1985.0067

Kremkow, J., Aertsen, A., and Kumar, A. (2010a). Gating of signal propagation in spiking neural networks by balanced and correlated excitation and inhibition. J. Neurosci. 30, 15760-15768. doi: 10.1523/JNEUROSCI.3874 $-10.2010$

Kremkow, J., Perrinet, L. U., Masson, G. S., and Aertsen, A. (2010b). Functional consequences of correlated excitatory and inhibitory conductances in cortical networks. J. Comput. Neurosci. 28, 579-594. doi: 10.1007/s10827-0100240-9

Kuffler, S. W. (1953). Discharge patterns and functional organization of mammalian retina. J. Neurophysiol. 16, 37-68. doi: 10.1152/jn.1953.16.1.37

Lam, Y. W., Cox, C. L., Varela, C., and Sherman, S. M. (2005). Morphological correlates of triadic circuitry in the lateral geniculate nucleus of cats and rats. J. Neurophysiol. 93, 748-757. doi: 10.1152/jn.00256.2004

Lapicque, L. (1907). Recherches quantitatives sur l'excitation électrique des nerfs traitée comme une polarization. J. Physiol. Pathol. Gen. 9, 620-635.

Lisman, J. E., and Idiart, M. A. (1995). Storage of $7+/-2$ short-term memories in oscillatory subcycles. Science 267, 1512-1515. doi: 10.1126/science.78 78473

Lisman, J. E., and Jensen, O. (2013). The theta-gamma neural code. Neuron 77, 1002-1016. doi: 10.1016/j.neuron.2013.03.007

McCormick, D. A., Connors, B. W., Lighthall, J. W., and Prince, D. A. (1985). Comparative electrophysiology of pyramidal and sparsely spiny stellate neurons of the neocortex. J. Neurophysiol. 54, 782-806. doi: 10.1152/jn.1985. 54.4 .782

Meng, X., Huguet, G., and Rinzel, J. (2012). Type III excitability, slope sensitivity and coincidence detection. Discrete \& Continuous Dynamical Syst. Ser. A 32, 2729-2757. doi: 10.3934/dcds.2012.32.2729

Miller, P. (2016). Dynamical systems, attractors and neural circuits. F1000Res. 5:F1000 Faculty Rev-992. doi: 10.12688/f1000research.7698.1

Miller, P., Brody, C. D., Romo, R., and Wang, X. J. (2003). A recurrent network model of somatosensory parametric working memory in the prefrontal cortex. Cereb. Cortex 13, 1208-1218. doi: 10.1093/cercor/bhg101

Mittmann, W., Koch, U., and Hausser, M. (2005). Feed-forward inhibition shapes the spike output of cerebellar Purkinje cells. J. Physiol. 563, 369-378. doi: 10.1113/jphysiol.2004.075028

Morgan, J. L., and Lichtman, J. W. (2020). An individual interneuron participates in many kinds of inhibition and innervates much of the mouse visual thalamus Neuron 106, 468-481.e2. doi: 10.1016/j.neuron.2020.02.001

Movshon, J. A., Kiorpes, L., Hawken, M. J., and Cavanaugh, J. R. (2005). Functional maturation of the macaque's lateral geniculate nucleus. J. Neurosci. 25, 2712-2722. doi: 10.1523/JNEUROSCI.2356-04.2005 
Murphy, B. K., and Miller, K. D. (2009). Balanced amplification: a new mechanism of selective amplification of neural activity patterns. Neuron 61, 635-648. doi: 10.1016/j.neuron.2009.02.005

Nakamura, P. A., and Cramer, K. S. (2011). Formation and maturation of the calyx of Held. Hear. Res. 276, 70-78. doi: 10.1016/j.heares.2010.11.004

Porter, J. T., Johnson, C. K., and Agmon, A. (2001). Diverse types of interneurons generate thalamus-evoked feedforward inhibition in the mouse barrel cortex. J. Neurosci. 21, 2699-2710. doi: 10.1523/JNEUROSCI.21-08-02699.2001

Pouille, F., Marin-Burgin, A., Adesnik, H., Atallah, B. V., and Scanziani, M. (2009). Input normalization by global feedforward inhibition expands cortical dynamic range. Nat. Neurosci. 12, 1577-1585. doi: 10.1038/nn.2441

Pouille, F., and Scanziani, M. (2001). Enforcement of temporal fidelity in pyramidal cells by somatic feed-forward inhibition. Science 293, 1159-1163. doi: 10.1126/science.1060342

Rock, C., and Apicella, A. J. (2015). Callosal projections drive neuronalspecific responses in the mouse auditory cortex. J. Neurosci. 35, 6703-6713. doi: 10.1523/JNEUROSCI.5049-14.2015

Schnupp, J. W., and Carr, C. E. (2009). On hearing with more than one ear: lessons from evolution. Nat. Neurosci. 12, 692-697. doi: 10.1038/nn.2325

Seung, H. S., Lee, D. D., Reis, B. Y., and Tank, D. W. (2000). Stability of the memory of eye position in a recurrent network of conductance-based model neurons. Neuron 26, 259-271. doi: 10.1016/s0896-6273(00)81155-1

Shadlen, M. N., and Newsome, W. T. (1998). The variable discharge of cortical neurons: implications for connectivity, computation and information coding. J. Neurosci. 18, 3870-3896. doi: 10.1523/JNEUROSCI.18-10-038 70.1998

Sherwin, J., Muraskin, J., and Sajda, P. (2012). You can't think and hit at the same time: neural correlates of baseball pitch classification. Front. Neurosci. 6:177. doi: 10.3389/fnins.2012.00177

Soltesz, I., and Crunelli, V. (1992). GABAA and pre- and post-synaptic GABAB receptor-mediated responses in the lateral geniculate nucleus. Prog. Brain Res. 90, 151-169. doi: 10.1016/s0079-6123(08)63613-4

Stratford, K. J., Tarczy-Hornoch, K., Martin, K. A. C., Bannister, N. J., and Jack, J. J. B. (1996). Excitatory synaptic inputs to spiny stellate cells in cat visual cortex. Nature 382, 258-261. doi: 10.1038/382258a0

Suzuki, S., and Rogawski, M. A. (1989). T-type calcium channels mediate the transition between tonic and phasic firing in thalamic neurons. Proc. Natl. Acad. Sci. U S A 86, 7228-7232. doi: 10.1073/pnas.86.18.7228

Swadlow, H. A. (2002). Thalamocortical control of feed-forward inhibition in awake somatosensory 'barrel' cortex. Philos. Trans. R. Soc. Lond. B Biol. Sci. 357, 1717-1727. doi: 10.1098/rstb.2002.1156

Swadlow, H. A. (2003). Fast-spike interneurons and feedforward inhibition in awake sensory neocortex. Cereb. Cortex 13, 25-32. doi: 10.1093/cercor/13.1.25

Swadlow, H. A., Bezdudnaya, T., and Gusev, A. G. (2005). Spike timing and synaptic dynamics at the awake thalamocortical synapse. Prog. Brain Res. 149, 91-105. doi: 10.1016/S0079-6123(05)49008-1

Swadlow, H. A., and Gusev, A. G. (2000). The influence of single VB thalamocortical impulses on barrel columns of rabbit somatosensory cortex. J. Neurophysiol. 83, 2802-2813. doi: 10.1152/jn.2000.83.5.2802

Swadlow, H. A., Gusev, A. G., and Bezdudnaya, T. (2002). Activation of a cortical column by a thalamocortical impulse. J. Neurosci. 22, 7766-7773. doi: 10.1523/JNEUROSCI.22-17-07766.2002

Thompson, S. M. (1994). Modulation of inhibitory synaptic transmission in the hippocampus. Prog. Neurobiol. 42, 575-609. doi: 10.1016/0301-0082(94) 90044-2

Thorpe, S., Fize, D., and Marlot, C. (1996). Speed of processing in the human visual system. Nature 381, 520-522. doi: 10.1038/381520a0

Tsodyks, M. V., and Markram, H. (1997). The neural code between neocortical pyramidal neurons depends on neurotransmitter release probability. Proc. Natl. Acad. Sci. U S A 94, 719-723. doi: 10.1073/pnas.94.2.719

Tsodyks, M. V., and Sejnowski, T. (1995). Rapid state switching in balanced cortical network models. Network 6, 111-124. doi: 10.1088/0954898X_6_2_001

Usrey, W. M., Reppas, J. B., and Reid, R. C. (1998). Paired-spike interactions and synaptic efficacy of retinal inputs to the thalamus. Nature 395, 384-387. doi: $10.1038 / 26487$

Van Hooser, S. D., Heimel, J. A., and Nelson, S. B. (2003). Receptive field properties and laminar organization of lateral geniculate nucleus in the gray squirrel (Sciurus carolinensis). J. Neurophysiol. 90, 3398-3418. doi: 10.1152/jn. 00474.2003

Van Hooser, S. D., Roy, A., Rhodes, H. J., Culp, J. H., and Fitzpatrick, D. (2013). Transformation of receptive field properties from lateral geniculate nucleus to superficial V1 in the tree shrew. J. Neurosci. 33, 11494-11505. doi: 10.1523/JNEUROSCI.1464-13.2013

van Rossum, M. C. W., Turrigiano, G. G., and Nelson, S. B. (2002). Fast propagation of firing rates through layered networks of noisy neurons. J. Neurosci. 22, 1956-1966. doi: 10.1523/JNEUROSCI.22-05-01956.2002

van Vreeswijk, C., and Sompolinsky, H. (1996). Chaos in neuronal networks with balanced excitatory and inhibitory activity. Science 274, 1724-1726. doi: $10.1126 /$ science.274.5293.1724

van Vreeswijk, C., and Sompolinsky, H. (1998). Chaotic balanced state in a model of cortical circuits. Neural Comput. 10, 1321-1371. doi: 10.1162/089976698300017214

Varela, J. A., Sen, K., Gibson, J., Fost, J., Abbott, L. F., and Nelson, S. B. (1997). A quantitative description of short-term plasticity at excitatory synapses in layer $2 / 3$ of rat primary visual cortex. J. Neurosci. 17, 7926-7940. doi: 10.1523/JNEUROSCI.17-20-07926.1997

Vogels, T. P., and Abbott, L. F. (2009). Gating multiple signals through detailed balance of excitation and inhibition in spiking networks. Nat. Neurosci. 12, 483-491. doi: 10.1038/nn.2276

Vogels, T. P., Sprekeler, H., Zenke, F., Clopath, C., and Gerstner, W. (2011). Inhibitory plasticity balances excitation and inhibition in sensory pathways and memory networks. Science 334, 1569-1573. doi: 10.1126/science.1211095

von Neumann, J. (1963). The Computer and the Brain. New Haven, CT: Yale University Press.

Wang, H. X., and Gao, W. J. (2009). Cell type-specific development of NMDA receptors in the interneurons of rat prefrontal cortex. Neuropsychopharmacology 34, 2028-2040. doi: 10.1038/npp.2009.20

Wehr, M., and Zador, A. M. (2003). Balanced inhibition underlies tuning and sharpens spike timing in auditory cortex. Nature 426, 442-446. doi: $10.1038 /$ nature 02116

Wehr, M., and Zador, A. M. (2005). Synaptic mechanisms of forward suppression in rat auditory cortex. Neuron 47, 437-445. doi: 10.1016/j.neuron.2005.06.009

Werblin, F. S. (2010). Six different roles for crossover inhibition in the retina: correcting the nonlinearities of synaptic transmission. Vis. Neurosci. 27, 1-8. doi: 10.1017/S0952523810000076

Wolf, J. A., Moyer, J. T., Lazarewicz, M. T., Contreras, D., Benoit-Marand, M., O’Donnell, P., et al. (2005). NMDA/AMPA ratio impacts state transitions and entrainment to oscillations in a computational model of the nucleus accumbens medium spiny projection neuron. J. Neurosci. 25, 9080-9095. doi: 10.1523/JNEUROSCI.2220-05.2005

Yang, W., Carrasquillo, Y., Hooks, B. M., Nerbonne, J. M., and Burkhalter, A. (2013). Distinct balance of excitation and inhibition in an interareal feedforward and feedback circuit of mouse visual cortex. J. Neurosci. 33, 17373-17384. doi: 10.1523/JNEUROSCI.2515-13.2013

Zhu, Y., Stornetta, R. L., and Zhu, J. J. (2004). Chandelier cells control excessive cortical excitation: characteristics of whisker-evoked synaptic responses of layer 2/3 nonpyramidal and pyramidal neurons. J. Neurosci. 24, 5101-5108. doi: 10.1523/JNEUROSCI.0544-04.2004

Conflict of Interest: The authors declare that the research was conducted in the absence of any commercial or financial relationships that could be construed as a potential conflict of interest.

Publisher's Note: All claims expressed in this article are solely those of the authors and do not necessarily represent those of their affiliated organizations, or those of the publisher, the editors and the reviewers. Any product that may be evaluated in this article, or claim that may be made by its manufacturer, is not guaranteed or endorsed by the publisher.

Copyright (c) 2022 Cao and Van Hooser. This is an open-access article distributed under the terms of the Creative Commons Attribution License (CC BY). The use, distribution or reproduction in other forums is permitted, provided the original author(s) and the copyright owner(s) are credited and that the original publication in this journal is cited, in accordance with accepted academic practice. No use, distribution or reproduction is permitted which does not comply with these terms. 